\title{
Influence of short rear end tapers on the wake of a simplified square-back vehicle: wake topology and rear drag
}

\author{
Anna-Kristina Perry ${ }^{1}\left[\right.$ Giancarlo Pavia $^{1} \cdot$ Martin Passmore $^{1}$
}

Received: 17 June 2016 / Revised: 3 October 2016 / Accepted: 4 October 2016 / Published online: 18 October 2016

(C) The Author(s) 2016. This article is published with open access at Springerlink.com

\begin{abstract}
As vehicle manufacturers work to reduce energy consumption of all types of vehicles, external vehicle aerodynamics has become increasingly important. Whilst production vehicle shape optimisation methods are well developed, the need to make further advances requires deeper understanding of the highly three-dimensional flow around bluff bodies. In this paper, the wake flow of a generic bluff body, the Windsor body, based on a square-back car geometry, was investigated by means of balance measurements, surface pressure measurements and 2D particle image velocimetry planes. Changes in the wake topology are triggered by the application of short tapers ( $4 \%$ of the model length) to the top and bottom edges of the base, representing a shape optimisation that is realistic for many modern production vehicles. The base drag is calculated and correlated with the aerodynamic drag data. The results not only show the effectiveness of such small devices in modifying the time average topology of the wake but also shed some light on the effects produced by different levels of upwash and downwash on the bi-stable nature of the wake itself.
\end{abstract}

\section{Introduction}

As progressively stricter $\mathrm{CO}_{2}$ regulations have come into effect in the last 30 years there has been a drive to improve vehicle powertrains, through downsizing, hybridisation and

Anna-Kristina Perry

A.Perry@lboro.ac.uk

Martin Passmore

M.A.Passmore@lboro.ac.uk

1 Loughborough University, Stewart Miller Building, Loughborough, Leicestershire LE11 3TU, UK electrification and to lower the overall energy consumption by reducing weight, tyre rolling losses and aerodynamic drag. The introduction of hybrids, fuel cells and fully electric powertrains introduces the additional problem of limited range so this further increases the relative importance of reducing sources of drag. The aerodynamic drag is the dominant source of losses for any ground vehicle above speeds of about $80 \mathrm{kph}$.

Square-back shapes are a popular design in the automotive industry due to their large cabin space and easy loading ability; however, the large base area and associated wake are responsible for a significant proportion of the aerodynamic drag. In steady state, the wake generated by such geometries has been shown to form a toroidal structure contained by four shear layers emanating from the trailing edges; Rouméas et al. (2009a) have demonstrated this in CFD and Perry et al. (2016) in a tomographic PIV investigation. This structure is the source of significant pressure loss and interacts with the main flow in the longitudinal direction forming a pair of counter-rotating vortices downstream of the near-wake region (Krajnovic and Davidson 2003).

It is widely accepted that changing the size and shape of this wake structure can increase the base pressure and thereby reduce the pressure drag. Many previous studies have demonstrated the effects of passive elements such as splitter plates and base cavities (Duell and George 1993), passive base bleed (Brown et al. 2010), base slats (Littlewood et al. 2011), forebody roughness (Van Raemdonck and Van Tooren 2008), underbody roughness (Perry and Passmore 2013) and base flaps (Grandemange et al. 2013c). Research has also been conducted into several active flow control techniques such as suction (Rouméas et al. 2009b), blowing (Littlewood and Passmore 2012), oscillated suction and blowing (Kim et al. 2004) and moveable vortex 
generators (Aider et al. 2010). Whatever the method of changing the wake, a better understanding of the wake topology and its link with the pressure distribution acting over the rear facing surfaces is important in order to maximise the benefits from any optimisation process.

Applying high aspect ratio tapers (representative of a production passenger car shape optimisation) to the roof trailing edge of a Windsor body, Littlewood and Passmore (2010) characterised the sensitivity of the wake of a squareback model to small changes in the trailing edge shape. The drag minimum occurred at a taper angle of $12^{\circ}$ from the horizontal with the reduction in the overall aerodynamic drag $(-2.7 \%)$ exceeding the reduction in the base area $(-1.1 \%)$. It was shown that the drag reduction arose from an increase in the base pressure but for taper angles greater than $12^{\circ}$ the increasing suction on the tapered surface outweighs the base pressure drag reduction and an increase in the drag was found.

Perry et al. (2015) applied similar tapers to both horizontal trailing edges of the same geometry. Taper angles of $0^{\circ}, 6^{\circ}, 12^{\circ}, 16^{\circ}$ and $20^{\circ}$ were considered and the different combinations of top and bottom tapers led to different levels of downwash or upwash in the near wake that resulted in a noticeable change in the base pressure distribution and therefore a variation of the base drag. In addition, using a similar approach to Grandemange et al. (2013c), a parabolic relationship was found between the drag and the lift data recorded for all 25 configurations tested:

$C_{D}=C_{D 0}+k\left(C_{L}-C_{L 0}\right)^{2}$

where $C_{D 0}$ and $C_{L 0}$ are the drag and lift coefficients related to the minimum drag configuration and $k$ is the gradient of the parabola, equal to 1.45 in this case. The data were then normalised using the optimum point on the parabola, corresponding to $\left(C_{D}-C_{D 0}\right)$ and $\left(C_{L}-C_{L 0}\right)$. It was found that all the data for $0^{\circ}-12^{\circ}$ top taper angles collapse onto a single curve; however, the data for the $16^{\circ}$ top taper angle lies slightly away from this trend line and the data for a $20^{\circ}$ top taper angle show no relation to the trend line. These anomalies were ascribed to the presence of a partially separated flow over the $16^{\circ}$ top taper and a fully separated flow over the $20^{\circ}$ top taper.

Although the analysis of time-averaged fields has proved to be very useful for wake topology characterisation, a better understanding of the effect of the wake on the base pressure can only be achieved by taking into account the unsteady features of the flow. Grandemange et al. (2013b) analysed the coherent dynamics of the wake of a 1/4 scale Ahmed body for a height-based Reynolds number equal to $9.2 \times 10^{4}$. The presence of two very distinctive time scales was found, respectively, at $T_{s} \sim 5 H / U_{0}$ and at $T_{l} \sim 10^{3} H / U_{0}$ (where $U_{0}$ is the free stream velocity and $H$ the model height). The shorter time scale relates to weak coherent oscillations of the wake in the vertical and lateral directions, associated with the interaction of the top-bottom and lateral shear layers, respectively, as seen in the work of Duell and George (1999). The longer time scale represents the random shift of the recirculation region between two symmetry-breaking positions, leading to a statistically symmetric wake. The sequence of these asymmetric states behaves like a stationary Markov chain and produces an unsteady side force that is thought to be responsible for part of the drag (Grandemange et al. 2013b).

Although this bi-stable behaviour seems to be independent of the value of $R e$, it is affected by the ground clearance and the base aspect ratio (Grandemange et al. 2013a). In fact, considering a modified version of the Ahmed body, it was proved that this particular type of instability vanishes for values of the non-dimensional ground clearance $C^{*}=C / W(C$ is the measured ground clearance and $W$ the model width) between 0.03 and 0.08 , due to the separation of the underbody flow on the ground. As aspect ratio was changed, the wake showed a bi-stable motion in the lateral direction, for values of model base width $W$ greater than model base height $H$, and in the vertical direction, when $H>W$. The wall proximity, however, does not necessarily stabilise the flow, since the wake showed a certain degree of bi-stability in the lateral direction even at zero ground clearance.

In addition Grandemange et al. (2014), again employing the Ahmed geometry, studied the sensitivity of the wake to vertical and horizontal control cylinders introduced to disturb the natural flow. They found that when such perturbation breaks the symmetry of the set-up, one of the two asymmetric topologies is selected, yielding a side force and therefore a drag increase. However, when the reflectional symmetry is preserved some, perturbations in the horizontal and vertical planes can alter the bi-stable nature of the wake yielding a drag reduction. In addition, it was found that the horizontal perturbation can affect the lift force, especially when the cylinder was used to control the top or the bottom mixing layer.

The stochastic nature of bi-stability together with its sensitivity to yaw angle was confirmed also by the results of Volpe et al. (2015), considering a quarter-scale Ahmed body for two height-based Reynolds numbers, $R e_{H}=5.1 \times 10^{5}$ and $R e_{H}=7.7 \times 10^{5}$. Furthermore, they highlighted the effects produced by the bi-stable behaviour of the wake on the base pressure distribution, isolating the two different states by conditional averaging the recorded velocity and pressure data. Similar results were obtained by Pavia et al. (2016) using surface pressure measurements on the base of a Windsor body with different trailing edge tapers.

The time-dependent structure of these bi-stable wake modes was investigated by Evrard et al. (2016), who 
proposed that when a square-back Ahmed model experiences bi-stability each reflectional symmetry-breaking mode is a horseshoe vortex and it is the temporal average of these asymmetric structures that results in the toroidal vortex structure that is traditionally reported in vehicle aerodynamics. This, however, is not the structure that is proposed by Volpe et al. (2015), who reconstructed the wake in each of the bi-stable modes behind an Ahmed body using spatial conditional averaging of multiple planes of 2D two component PIV data, and concluded that the overall wake structure remained a torus but biased towards a dominant side.

This work aims to asses the presence of bi-stability in the wake of a vehicle-like geometry (the Windsor body). The sensitivity of this phenomenon is then studied through the application of short rear end tapers, which are typically used in the automotive industry for drag optimisation. This was achieved through balance and surface pressure measurements as well as 2D planar particle image velocimetry (PIV).

\section{Experimental methodology}

\subsection{The Windsor body}

The Windsor body employed in the work reported here has been considered by several authors such as Littlewood and Passmore (2010) and Perry et al. (2015). The model is a simplified geometry designed to generate the flow structures of a typical passenger car. The slanted front end is more representative than the more widely used Ahmed body, and it also lends itself to a wider range of rear end modifications. It is also more representative when used at yaw. At the scale used in this work (Length $L=1044 \mathrm{~mm}$, width $W=389 \mathrm{~mm}$, height $H=289 \mathrm{~mm}$ ), the model is approximately equivalent to a $1 / 4$ scale passenger car, giving a tunnel blockage of $4.4 \%$, and it is tested at a ground clearance of $50 \mathrm{~mm}(17.3 \%$ of the model height).

The Windsor body used for this study has a removable rear section that allows testing of multiple rear taper angles. Each taper has a chord $c$ of $45 \mathrm{~mm}$ giving a fixed aspect ratio AR of 8.6 (AR defined as the ratio of the model width to chord of the taper); slant angles of $0^{\circ}, 6^{\circ}, 12^{\circ}, 16^{\circ}$, and $20^{\circ}$ have been considered. Three cases have been studied, based on the results of Perry et al. (2015): in the first two cases, a slant was applied only to the upper or lower horizontal trailing edges, leaving the opposite square, whilst a third set of tests was performed applying a $12^{\circ}$ taper to the bottom edge of the base whilst varying the slant at the top.

For the sake of clarity, $\phi_{t}$ denotes the angle formed by the top taper with the flat section of model roof, whereas $\phi_{b}$

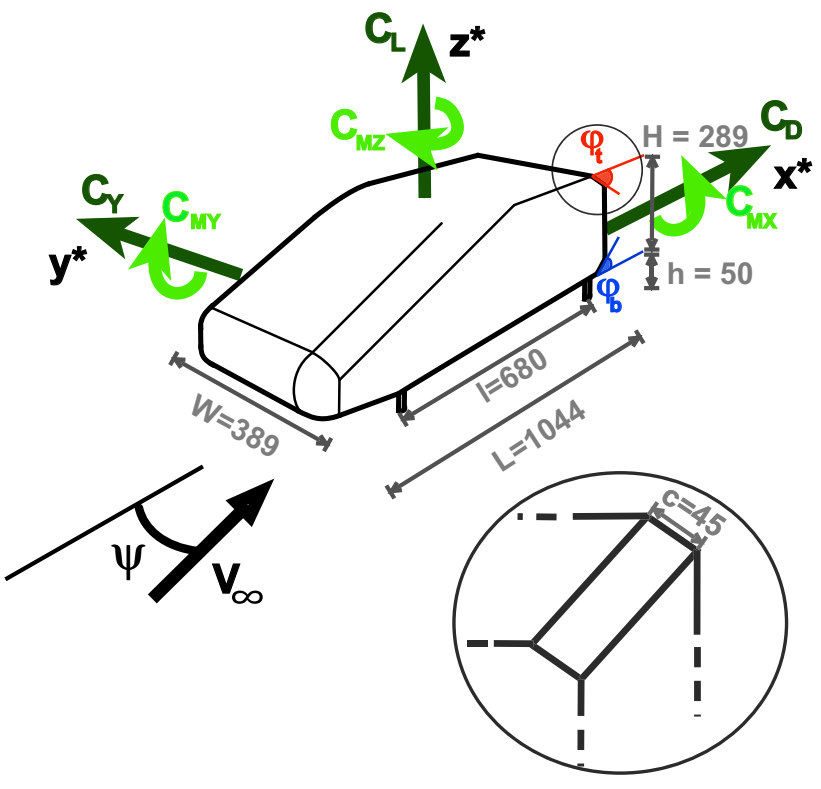

Fig. 1 Schematic representation of the model considered. All measures are expressed in $\mathrm{mm}$. For the reference system see (SAE 2010)

represents the angle between the bottom chamfer and the flat model underbody (see Fig. 1).

The model is mounted via four pins (M8 threaded bar) connected to the six-component balance located beneath the working section floor. The pins were in locations representative of the front and rear axles and $10 \mathrm{~mm}$ inboard of the model sides. The SAE coordinate system (SAE 2010) is used throughout; the $X$ axis is aligned with the flow in the downstream direction, the $Z$ axis is vertical, positive upwards, and the $Y$ axis follows a right-handed coordinate system. The origin is on the ground plane at mid-wheelbase, mid-track (Fig. 1).

All the dimensions as well as the coordinates in the reference systems have been normalised using the model height $H$ as the reference length and are denoted with the superscript $*$ throughout the paper. In a similar way, the velocity magnitude has been normalised with the free stream value $V_{\infty}$ and is labelled as $V^{*}$.

\subsection{The wind tunnel}

All experiments were carried out in the Loughborough University large wind tunnel, Johl (2010), and all tests were performed with a free stream velocity of $40 \mathrm{~m} / \mathrm{s}$, corresponding to a Reynolds number $R e_{H}$ of $7.7 \times 10^{5}$ based on the model height. The working section is $1.92 \times 1.32 \times 3.6 \mathrm{~m}\left(W_{T} \times H_{T} \times L_{T}\right)$ with a fixed floor and no upstream boundary layer treatment. In empty conditions, the free stream turbulence level inside the test section is approximately $0.2 \%$, with a flow uniformity of $\pm 0.4 \%$ 
of the mean flow value. In this state, the boundary layer thickness at the model origin is $\delta_{99}=64 \mathrm{~mm}$.

\subsection{Balance measurements}

The aerodynamic loads were recorded by means of an Aerotech six-component virtual centre balance, located under the working section of the tunnel. It features analogue to digital conversion at the load cell to minimise signal degradation, and an automated yaw mechanism with a positional accuracy of $0.1^{\circ}$. Further information can be found in Johl (2010). The aerodynamic loads were sampled at $100 \mathrm{~Hz}$ for $150 \mathrm{~s}$ or $630 \mathrm{~s}$, depending on the test. Before starting to $\log$ the data, a $30 \mathrm{~s}$ settling time was used for all measurements. All forces and moments were non-dimensionalised using Eqs. 2 and 3:

$C_{\mathrm{Fi}}=\frac{\text { Force }}{0.5 \rho \mathrm{SV}_{\infty}^{2}}$

$C_{\mathrm{Mi}}=\frac{\text { Moment }}{0.5 \rho \mathrm{SV}_{\infty}^{2} l}$

where $\rho$ is the air density, $S$ is the projected model frontal area $\left(S=0.1124 \mathrm{~m}^{2}\right), V_{\infty}$ is the free stream velocity and $l=680 \mathrm{~mm}$ the model wheelbase (equal to the longitudinal distance between the pins connecting the model to the balance). All coefficients were corrected for blockage effects using Eq. 4:

$C_{\mathrm{Fi}_{\mathrm{Cor}}}=C_{\mathrm{Fi}}(1-B)^{2}$

where $B$ denotes the blockage value, given by the ratio between the model frontal area $S$ and the tunnel working section cross-sectional area $S_{T}$.

\subsection{Pressure measurements}

The pressure acting on the model rear facing surfaces was recorded by populating the entire base and tapers with a grid of pressure tappings connected via flexible tubes to a pair of Chell CANdaq miniature pressure scanners, with a manufacturer quoted accuracy of $\pm 1.47 \mathrm{~Pa}$, mounted inside the model. Each scanner consists of 64 piezoresistive pressure sensors, paired with temperature sensors to allow the correction of inaccuracies introduced by temperature drift. The tappings were placed with a finer distribution close to the model edges, to get a more accurate representation of the pressure distribution in the regions with the highest gradients (see Fig. 2); 72 taps were used for the model base and 24 for each slanted surface. Pressure data were recorded at $260 \mathrm{~Hz}$ for $630 \mathrm{~s}$; the long sample time was required to obtain a statistically symmetric average (Pavia et al. 2016). The free stream dynamic and static pressures were recorded $1870 \mathrm{~mm}$ upstream of the model, at the start

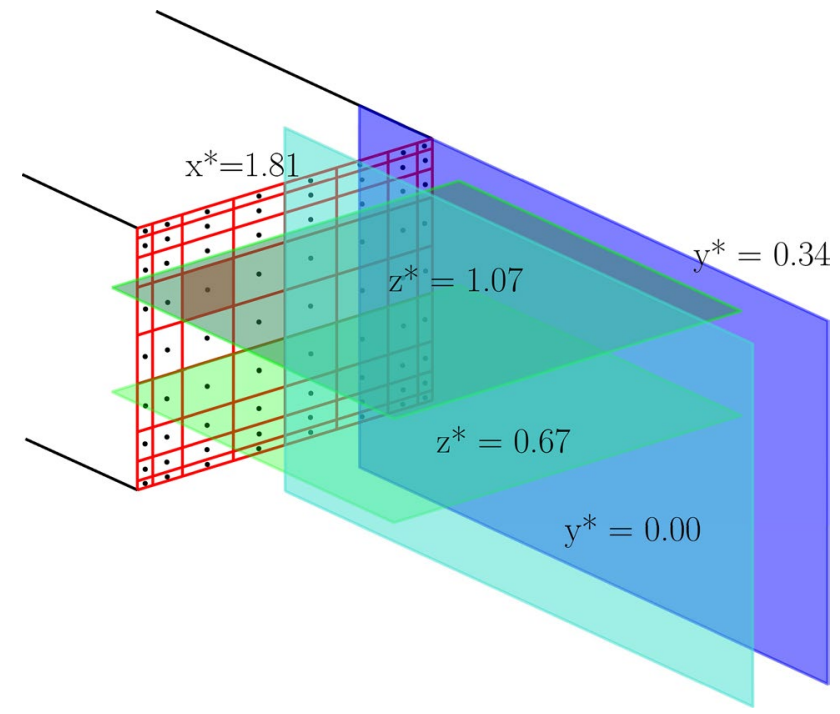

Fig. 2 Representation of the model base with the pressure tappings and the 2D PIV planes considered in the present work. The red square on the model base denotes the area associated with the $n^{\text {th }}$ tap used for the estimation of the area weighted drag

of the working section. Once the pressure coefficients were calculated, Eq. 5, the results were corrected for blockage using the MIRA correction (based on continuity), Eq. 6:

$C_{P}=\frac{p-p_{\infty}}{0.5 \rho \mathrm{SV}_{\infty}^{2}}$

$1-C_{P_{\text {corr }}}=\left(1-C_{\mathrm{p}}\right)(1-B)^{2}$

where $p_{\infty}$ is the free stream static pressure. The contribution of the model rear facing surfaces to the aerodynamic force experienced by the model was estimated by integrating the measured pressure field:

$C_{D_{\text {Rear }}}=-\frac{1}{S} \iint_{S} C_{\mathrm{p}} \cdot \mathrm{d} S \simeq-\frac{1}{S} \sum_{i=1}^{N_{\text {tap }}} C_{p_{i}} S_{i}$,

where $C_{p_{i}}$ is the time-averaged value of the pressure coefficient recorded by the $n^{\text {th }}$ tap, and $S_{i}$ is the projection of the associated area (see Fig. 2).

Since the flow field analysed in this study is highly sensitive to any asymmetry present in the experimental setup the model was yawed to the onset flow until the most symmetric base pressure distribution was achieved over the long sample time of $630 \mathrm{~s}$, and this was assumed to be where the model axis and onset flow axis were aligned.

\subsection{PIV measurements}

Two-dimensional PIV fields were taken on four orthogonal planes using a LaVision system consisting of two CCD dual 
frame 4 Mpixels cameras, a $200 \mathrm{~mJ}$ double pulsed Nd:YAG laser and a PIVTec 45 atomiser, generating $1 \mu \mathrm{m}$ droplets of DEHS. Two vertical and two horizontal streamwise planes were considered. The first vertical plane $\left(y^{*}=0\right)$ was placed along the model centreline, whilst the second plane $\left(y^{*}=0.34\right)$ was located at $1 / 4$ of the model width (on the right hand side of the model), where $y^{*}$ is the distance of the plane from model centreline normalised with the model height $H$. In a similar way, the first horizontal plane $\left(z^{*}=0.67\right)$ was placed at the middle of the model base, whilst the second plane $\left(z^{*}=1.07\right)$ was located $30 \mathrm{~mm}$ down from the top trailing edge, where $z^{*}$ is the non-dimensional distance of each plane from the ground (see Fig. 2). For measurements on the vertical planes, two cameras were placed next to each other, giving an $800 \times 400 \mathrm{~mm}(l \times h)$ field of view, with a $50 \mathrm{~mm}$ overlapping region. For the horizontal streamwise planes, a single camera was used, giving a $400 \times 400 \mathrm{~mm}$ field of view.

During each acquisition, 1000 image pairs were captured to ensure reasonable confidence in the mean velocity across all areas of the field of view (Passmore et al. 2010). A capture rate of $7.26 \mathrm{~Hz}$ was used, resulting in an acquisition time of $137.7 \mathrm{~s}$. The data were then post-processed using DaVis 8.2.0, applying a multi-pass scheme for crosscorrelation starting with an interrogation window with a size of $64 \times 64$ pixels and a $50 \%$ overlap between cells and ending with windows of $32 \times 32$ pixels and a $75 \%$ overlap, yielding a final spatial resolution of $4 \times 10^{-6} \mathrm{~m}^{2}$. Due to limited optical access during the PIV data acquisition, the model was moved forward in the working section and fixed to the tunnel floor and therefore it was aligned to the tunnel axis rather than the onset flow axis.

\section{Results and discussion}

\subsection{Square-back configuration}

The time-averaged results obtained for the square-back configuration are presented in Fig. 3. The pressure map shows the existence of a relatively high-pressure region slightly below the vertical centre of the base, surrounded by a lower pressure area. This lower pressure region covers most of the base and its shape confirms the existence of the toroidal vortex structures described by Rouméas et al. (2009a) for the Ahmed body with similar rear end geometry. The structure is confirmed in the vertical PIV plane at $y^{*}=0.00$ (Fig. 3), that is characterised by the presence of two almost equal recirculating regions, with the top portion of the vortex slightly larger than the bottom due to the combined effects of the fore-body slant and the ground proximity. The free stagnation point is located at $x^{*}=1.58$ downstream of the model base and is aligned with the rear

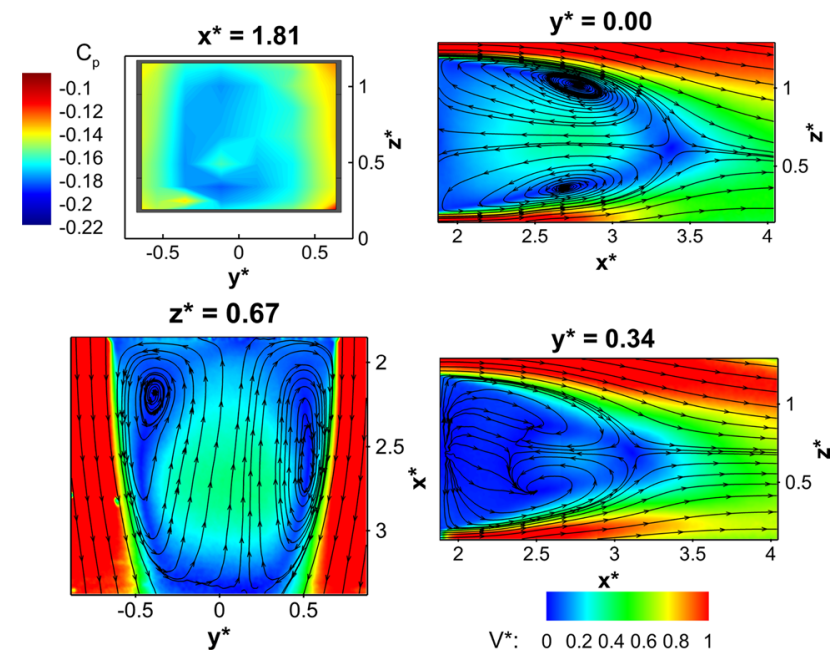

Fig. 3 Time-averaged results for the square-back Windsor body. Clockwise from top left: $x^{*}=1.81$ base pressure distribution, $y^{*}=0.00$ PIV vertical mid-plane, $y^{*}=0.34$ PIV vertical off-centre plane, $z^{*}=0.67$ PIV horizontal mid-plane

stagnation point at $z^{*}=0.6$, justifying the increase in the static pressure previously described in this region as the result of the impingement of the flow on the model rear end.

The interaction between these two vortical structures leads to a local acceleration of the reverse flow in the central region of the near wake, with velocities of $40-60 \%$ of the free stream value, before slowing down in proximity to the base itself.

Similar features are evident on the mid-horizontal plane (Fig. 3, $z^{*}=0.67$ ), where the two lobes of the wake torus are still recognisable. However, the cores of the two recirculating regions are now closer to the model base, with the closest being at $x^{*}=0.39$ from the base $\left(x^{*}=0.88\right.$ for the mid-vertical plane), explaining the stronger pressure gradient that characterises the time average pressure map in the horizontal direction. The two horizontal lobes, though, are not the same shape, with the core of the recirculating region almost circular on the left hand side and elliptical on the right, and the reverse flow impinging on the base does not seem to be aligned with the rear stagnation point previously described. The topology of this flow field resembles one of the two reflectional symmetry-breaking modes shown in previous studies such as Grandemange et al. (2013b) and Volpe et al. (2015). The characteristic time between switches has been reported to be typically greater than $10 \mathrm{~s}$ (Volpe et al. 2015 and Pavia et al. 2016) which can lead to bias in the data when the sampling time is not sufficiently long (Grandemange et al. 2013b). This is the case for the PIV data acquired in the present work, as the sampling time was limited to $137.7 \mathrm{~s}$ for constant frequency data collection. Such constraint was not present during the pressure 
data acquisition; therefore, sufficient data were captured to obtain a statistically symmetric average. The two reflectional symmetry-breaking states were isolated by means of proper orthogonal decomposition (POD). This technique, proposed by Lumley (1967), allows one to represent a generic instantaneous field $F(\mathbf{x}, t)$ by considering a decomposition of the fluctuating part:

$\mathbf{F}(\mathbf{x}, t)=\mathbf{F}_{0}(\mathbf{x})+\mathbf{f}^{\prime}(\mathbf{x}, t)=\mathbf{F}_{0}(\mathbf{x})+\sum_{n=1}^{N} a_{n}(t) \phi_{n}(\mathbf{x})$,

where $N$ is the number of acquisitions, $\mathbf{F}_{0}$ and $\mathbf{f}^{\prime}$ the mean and the fluctuating components of the considered field, $\phi_{n}(\mathbf{x})$ the basis functions and $a_{n}(t)$ the related temporal coefficients (uncorrelated in time). More details about the estimation of the spatial functions and the temporal coefficient can be found in Sirovich (1987).

In the work reported here, POD was applied to each data collection technique. The probability density function (PDF) distributions related to the first temporal coefficient extracted from the pressure field and all the PIV planes for the square-back configuration are presented in Fig. 4. In all the cases, except the vertical mid-plane $\left(y^{*}=0.00\right)$, the existence of a bimodal distribution is shown, confirming the existence of two different states as previously shown in the findings of Grandemange et al. (2013b) and Volpe et al. (2015). To isolate the two states, a low order model was then constructed from the time-averaged field and the first mode according to Eq. 9, following a similar approach to Thacker et al. (2010):

$\mathbf{F}^{\mathrm{LOM}}(\mathbf{x}, t)=\mathbf{F}_{0}(\mathbf{x})+a_{1}(t) \phi_{1}(\mathbf{x})$

The realisations $\mathbf{F}^{\mathrm{LOM}}(\mathbf{x}, t)$ obtained were sorted according to the value of the first temporal coefficient $a_{1}(t)$ and filtered from the noise using a moving average filter with a cutoff frequency of $1 \mathrm{~Hz}$. The sorting criterion was based on the condition $a_{1}(t) \leq \tau$ or $a_{1}(t)>\tau$, where $\tau=\left(\max \left(a_{1}(t)\right)+\min \left(a_{1}(t)\right)\right) / 2$. The fields belonging to the same group were then averaged together, yielding the two different states presented in Fig. 5a, b.

The topology of the two isolated states, denoted here as $L$ State and $R$ State, appears to be in good agreement with results reported in the literature (Grandemange et al. 2013b; Evrard et al. 2016) (see Fig. 5a, b). Each state is characterised by the coexistence of two vortical structures (see Fig. 6a). The first is a vortex shed from one of the two vertical sides of the base, characterised by slow dynamics that results in the formation of a low pressure zone on the corresponding side of the base. The second structure is a "C" shaped horseshoe vortex, whose existence is suggested by the fact that the core of the smaller recirculation is visible on both of the conditionally averaged horizontal mid-planes (Fig. 5a, b at $z^{*}=0.67$ ) and is very similar in shape and streamwise position to the cores of the two recirculations captured in the time average vertical mid-plane (Fig. 3, $y^{*}=0.00$ ). These vortical structures are shed faster than the large horseshoe vortex; evidenced by the observation that this structure is positioned farther downstream from the base. This suggests a higher convective velocity and justifies the higher values of the static pressure obtained for the corresponding half of the base. This proposed topology appears to be in good agreement with the existence of the long-time and short-time wake dynamics proposed by Grandemange et al. (2013b).

These structures, however, disagree with that published by Evrard et al. (2016) who, without the information from the off-centre planes, speculated that the near wall vortex seen in the horizontal plane was connected with the vortices seen in the vertical mid-plane to create a single horseshoe vortex. With the additional information presented in this work, it is hypothesised that the near wall vortex is isolated as a transverse vortex, whilst all other vortex cores are connected in a "C" shaped vortex.
Fig. 4 Square-back Windsor body. a PDF distributions for the first POD temporal mode extracted from the base pressure data. b PDF distributions for the first POD temporal mode extracted from all the PIV planes considered in the present work: $z^{*}=0.67$ horizontal mid-plane, $z^{*}=1.07$ horizontal top plane, $y^{*}=0.00$ vertical mid-plane, $y^{*}=0.34$ vertical off-centre plane (a) 1st Temporal coeff. (Base pressure)

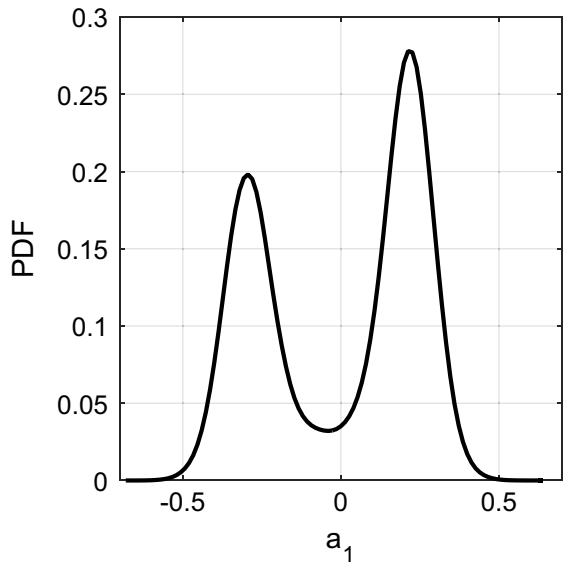

(b) 1st Temporal coeff. (PIV)

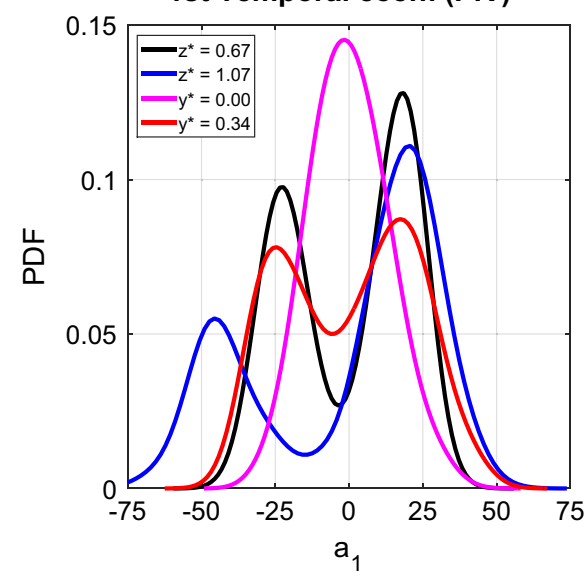



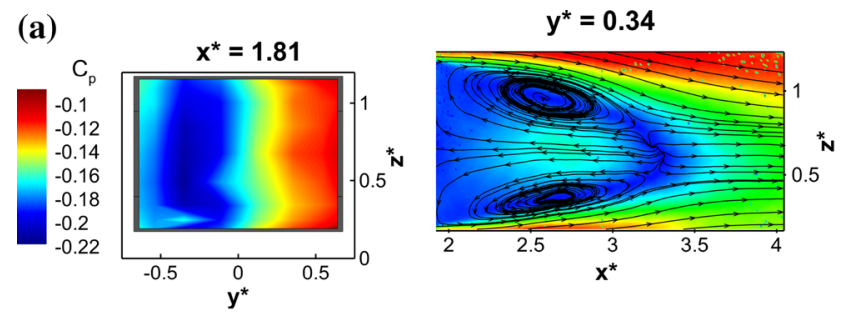

(b)
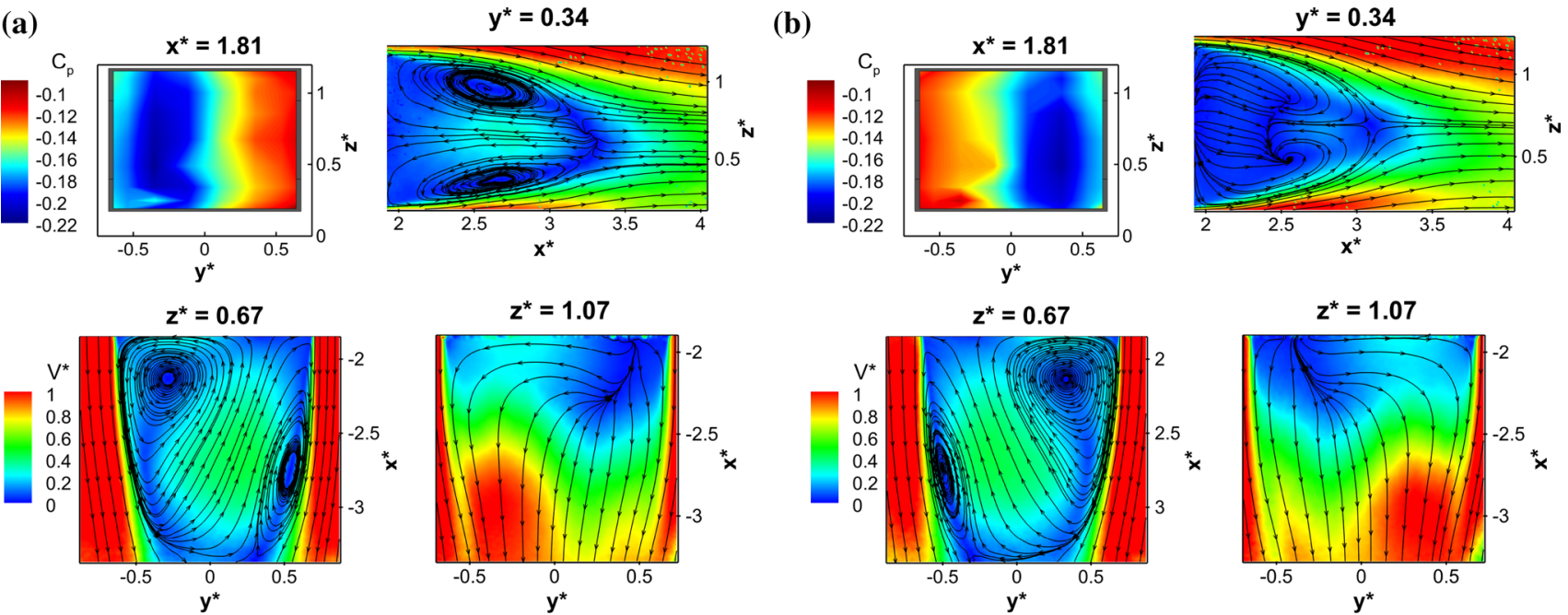

(c)
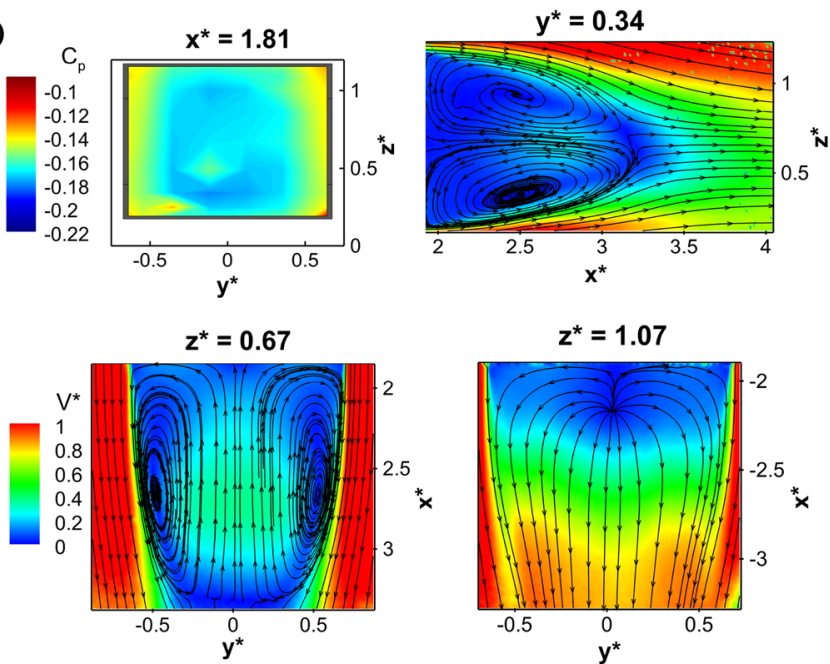

Fig. 5 Square-back Windsor body: a and b POD conditionally averaged states; $\mathbf{c}$ state averaged field. Clockwise from top left: $x^{*}=1.81$ base pressure distribution, $y^{*}=0.34$ PIV vertical off-centre plane,

$z^{*}=1.07$ PIV horizontal top plane, $z^{*}=0.67$ PIV horizontal midplane, a L state, $\mathbf{b}$ R state, $\mathbf{c}$ spatial average

Fig. 6 a Representation of one of the reflectional symmetrybreaking states, $\mathbf{b}$ representation of the wake torous (based on the PIV results)
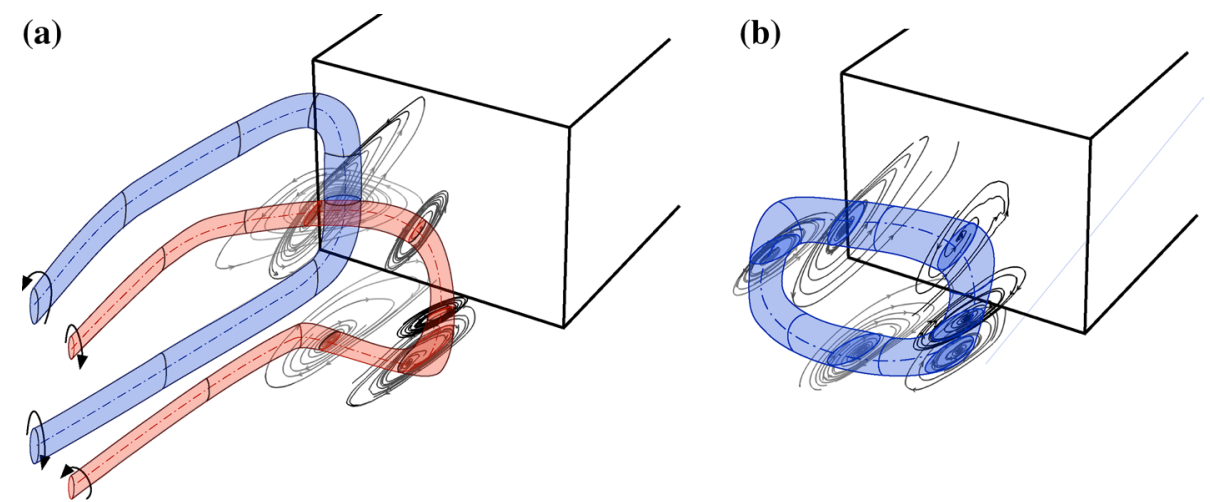

If the two modes generated in the POD conditional average are then averaged, Fig. $5 \mathrm{c}$, the velocity field produced is representative of a statistically balanced field (i.e. the sampling period $t \rightarrow \infty$ ); this is referred to here as the state average simply because it gives equal weight to the two states. From this a symmetric flow field is obtained in the 
two horizontal planes and an "open" wake structure is seen in the vertical off-centre plane with a clear bifurcation line between the two lobes of the vortex indicating that they are distinct and little flow travels between them. All these flow fields give merit to a toroidal wake structure (Fig. 6b) in agreement with the steady results of the simulations as reported by Krajnovic and Davidson (2003) and Rouméas et al. (2009a) for similar geometries, although this is a flow feature that is never present when the results are temporally resolved.

\subsection{Effects of bi-stability on side force, rolling moment and yawing moment}

To further investigate the effect of bi-stability balance results were taken with a focus on the lateral forces and moments. Data were recorded at $100 \mathrm{~Hz}$ for $630 \mathrm{~s}$ and the temporal trend was examined. The data recorded for the side force (Fig. 7a) resampled at $5 \mathrm{~Hz}$ shows a trend similar to that described in Volpe et al. (2015) and Pavia et al. (2016) for the temporal evolution of the static pressure

(a) Side Force Temporal Trend (Data resampled at $5 \mathrm{~Hz}$ )

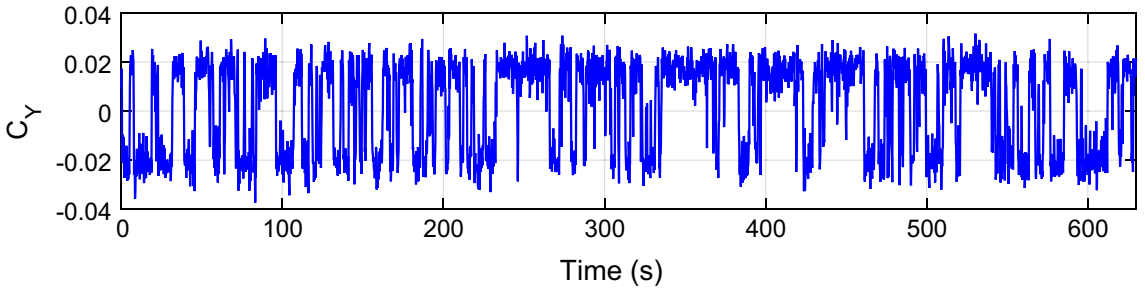

(b)

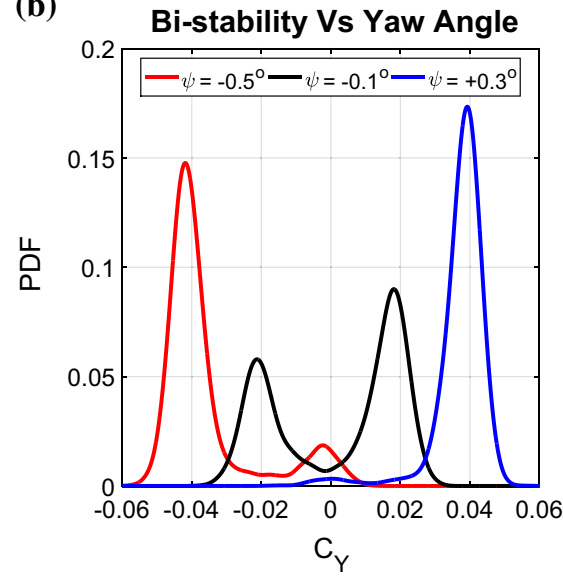

(c)

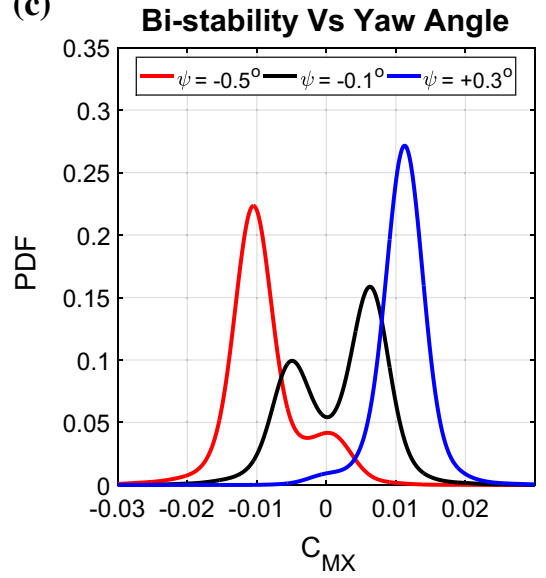

(d)
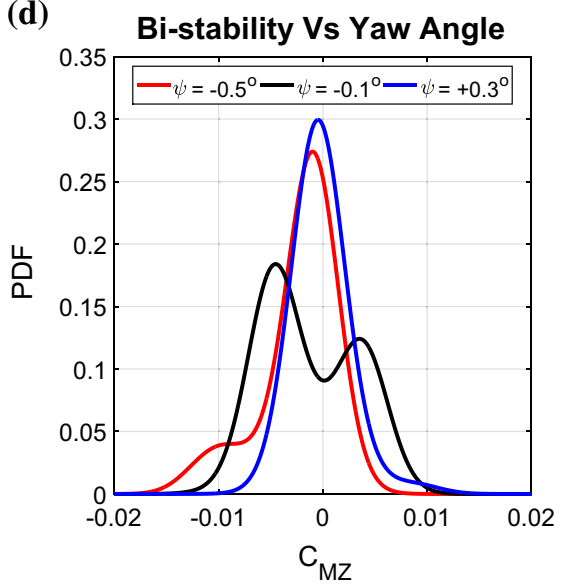

(e)

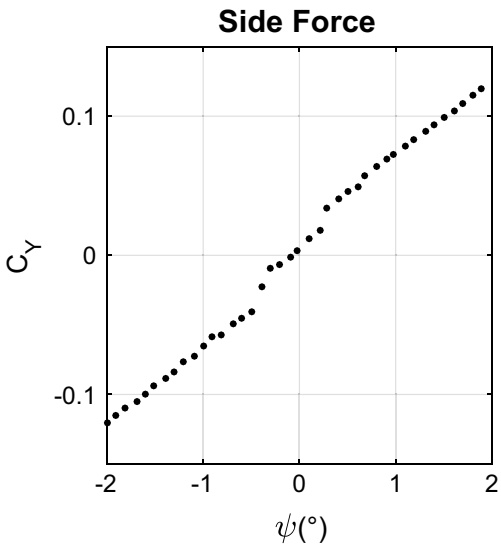

Fig. 7 a Side force temporal trend; b side force, c rolling moment and $\mathbf{d}$ yawing moment sensitivity to small variations of the model yaw angle (PDF distributions); time-averaged values of e side force, $\mathbf{f}$ (f)

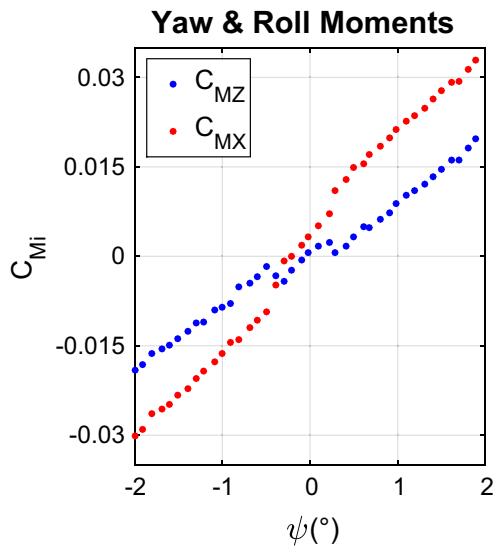

yawing and rolling moments recorded for the square-back configuration for $-2.0^{\circ}<\psi<2.0^{\circ}$ 
Fig. 8 a Drag and b lift recorded for all the tested configurations

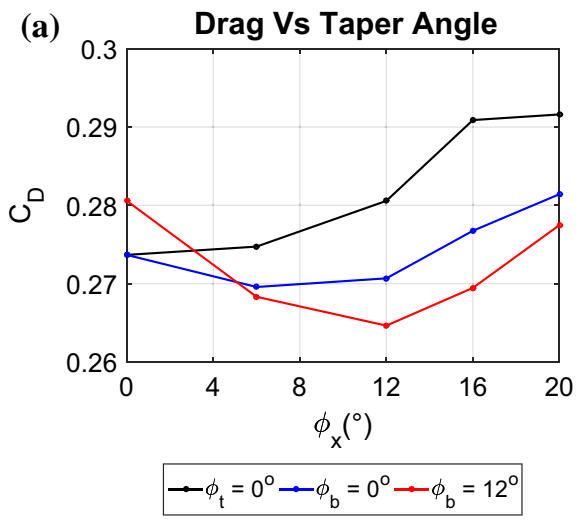

acting over the model base. The switch between the two different states results in the existence of two almost equiprobable values for each of the lateral components of the aerodynamic force, as shown by the bimodal distribution associated with the PDF distributions of the values of side force $C_{Y}$, rolling moment $C_{\mathrm{MX}}$ and yawing moment $C_{\mathrm{MZ}}$ recorded at $\psi=-0.1^{\circ}$ (Fig. $7 \mathrm{~b}-\mathrm{d}$ ). This behaviour, however, is very sensitive to small variations of the yaw angle (in good agreement with that found by Volpe et al. (2015) and Pavia et al. (2016) for the base pressure distribution and by Evrard et al. (2016) for the aerodynamic side force), as it disappears for $\psi \leq-0.5^{\circ}$ or $\psi \geq+0.3^{\circ}$, leading to the selection of one single state.

The extent of the bi-stable domain was then further investigated by performing a yaw sweep between $-2^{\circ}$ and $+2^{\circ}$ with steps of $0.1^{\circ}$. For each position, the values of the aerodynamic forces were recorded for $150 \mathrm{~s}$, with a settling time of $30 \mathrm{~s}$. The time-averaged results are presented in Fig. 7e, f. The plots clearly show the existence of a linear trend for $C_{Y}(\psi), C_{\mathrm{MX}}(\psi)$ and $C_{\mathrm{MZ}}(\psi)$, apart from $-0.5^{\circ}<\psi<+0.3^{\circ}$ where a nonlinear region can be seen due to the presence of the wake bi-stability.

\subsection{Taper effects}

The effects produced by the small tapers already described in Sect. 2.1 were then studied, focusing initially on their impact on the time-averaged wake topology and base pressure distribution as well as the steady aerodynamic forces acting over the entire model.

The balance results presented in Fig. 8 show that when the top angle is zero and the bottom taper angle is increased, the drag increases for all cases compared to the baseline square-back, reaching a maximum for $\phi_{b}=20^{\circ}$. As the bottom taper angle is increased the lift decreases to a minimum at $16^{\circ}$ before increasing for higher angles as the flow acting over the tapered surface becomes fully separated. If the upper taper is varied, whilst the lower slant is fixed at $0^{\circ}$ (as in the work of Littlewood and Passmore 2010), there is (a)

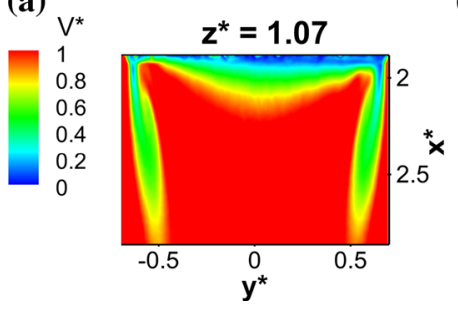

(b)

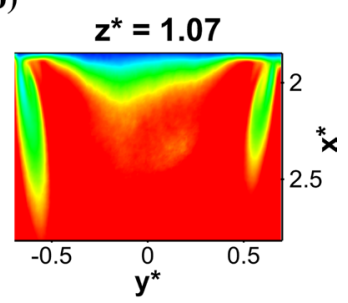

Fig. 9 Longitudinal tip vortices shed from the top trailing edge taper for two different top taper angles: $\mathbf{a} \phi_{t}=16^{\circ}, \mathbf{b} \phi_{t}=20^{\circ} ; \phi_{b}=0^{\circ}$ in both the cases

a small drag reduction compared to the baseline case as the taper angle is increased up to $12^{\circ}$, and then drag increases again, following a parabolic trend, becoming higher than in the square-back case for $\phi_{t} \geq 16^{\circ}$. The lift is the mirror image of the bottom taper data, with the maximum value of the lift recorded at $\phi_{t}=16^{\circ}$. The slope of the linear section of the curve, though, is now lower as the action of the slanted surface is partially counteracted by ground effect. With the lower taper angle fixed at $12^{\circ}$, the parabolic shape in the $C_{D}$ plot is preserved although with a steeper trend. In these conditions, a drag minimum is found when a $12^{\circ}$ taper is applied to the top trailing edge (low drag configuration). The lift curve, on the other hand, is characterised by the same trend already discussed for the $\phi_{b}=0^{\circ}$ case, although with a different value of $C_{L}$ for $\phi_{t}=0$.

The application of a chamfer to the horizontal edges of the model base creates a region of suction at the leading edge of the tapered surface. As the angle is increased the low pressure region tends to expand downstream until the flow starts to separate (usually at $\phi_{t, b}=20^{\circ}$ ), leading to a sudden reduction of the size of the low pressure region. The region of suction is accompanied by the formation of a pair of trailing vortices at the tip of the slant, seen in the PIV plane at $z^{*}=1.07$, close to the trailing edge of the top slant (see Fig. 9). These vortices are similar to those identified by Grandemange et al. (2013c) when applying flaps to the 
(a)

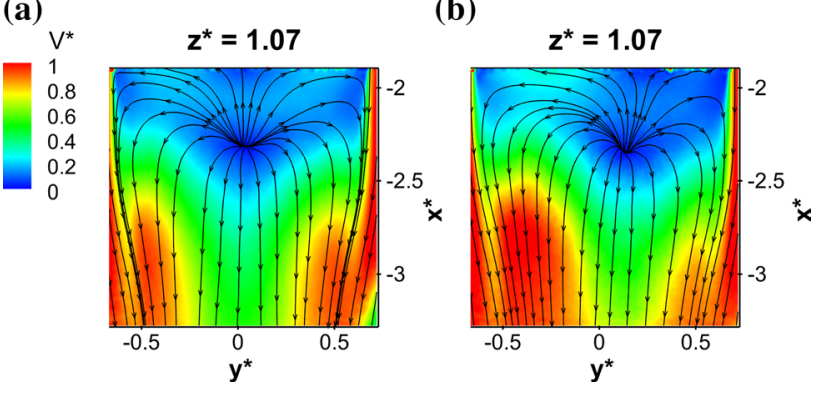

Fig. 10 Time-averaged PIV horizontal top plane: a $\phi_{t}=0^{\circ}$ and $\phi_{b}=12^{\circ}, \mathbf{b} \phi_{t}=0^{\circ}$ and $\phi_{b}=20^{\circ}$

top and bottom trailing edges of the Ahmed body. Once the flow over the slant is fully separated the length of the tip vortices is reduced and the flow field loses its left and right symmetry. This effect is shown in Fig. 9b where the flow close to the trailing edge of the top slant for $\phi_{t}=20^{\circ}$ is no longer symmetric. This asymmetry, however, is not limited to the slant region but seems to extend downstream over the entire wake of the model, as shown by the time-averaged PIV results presented in Fig. 10. In this case, the asymmetry triggered by the separation of the flow over the bottom slant (as in the case for $\phi_{b}=20$, Fig. 10b) is still captured by the PIV plane close to the model top trailing edge (at $z^{*}=1.07$ ), whilst a better symmetry can be seen for lower values of the diffuser angles (Fig. 10a).

The effects of the application of tapers to the bottom trailing edge on the time-averaged wake topology and the base pressure distribution are presented in Fig. 11a, b. Here, an increase in the taper angle results in an increase of the upwash, for example as seen on the PIV vertical midplane $\left(y^{*}=0.00\right.$ for $\phi_{t}=0^{\circ}$ and $\left.\phi_{b}=12^{\circ}\right)$. The saddle point that marks the wake closure tends to move upwards as the lower taper angle increases, reaching its maximum at $z^{*}=1.03$ for $\phi_{b}=16^{\circ}$; it then starts to move down as the flow over the bottom slant becomes fully separated. The streamlines leaving the saddle point are also angled at a similar trajectory, forming a maximum angle of almost $10^{\circ}$ for the configuration generating the lowest lift (downforce). The length of the recirculation zone is also reduced, being about $20 \%$ shorter than in the square-back case, whilst the symmetry previously seen between the top and bottom recirculations is lost. The high momentum flow passing underneath the model is deflected upwards by the slant and feeds the lower recirculating zone, increasing its size compared to the upper vortex. As a result, the direction of the flow impinging the base is tilted upwards forming an angle of about $108^{\circ}$ to the ground plane for $\phi_{b}=12^{\circ}$ and $\phi_{b}=16^{\circ}$. Consequently the base impingement point moves towards the top trailing edge and the annular low pressure region seen in the square-back case develops into a more
"U" shaped zone, extending throughout the lower portion and the sides of the base.

The PIV data acquired at $z^{*}=0.67$ show the effects of the upwash generated by the upswept slant. When the flow is attached to the bottom slant (e.g. Fig. 11a for the $\phi_{b}=12^{\circ}$ case) the symmetry of the wake, in the timeaveraged field, is restored. The two recirculation regions are pressed against the base and the streamlines close to the saddle point assume a characteristic convex shape. Once the flow over the slant becomes fully detached (Fig. 11b) the upwash is reduced, the near-wake separation becomes flatter, and a degree of asymmetry in the time-averaged flow field can be seen again. An additional consideration is that the increase in bottom chamfer angle can promote separation of the flow from the tunnel floor, for example at $x^{*}>2.5$, Fig. $11 \mathrm{a}, \mathrm{b}, y^{*}=0.00$. This region of separation, however, is quite limited and is sufficiently far from the model base that no significant effects are produced on either the base pressure distribution or the aerodynamic drag experienced by the model. The size of this separation zone tends to reduce in the spanwise direction and is hardly visible in the PIV data acquired at $y^{*}=0.34$.

The time-averaged velocity field in this last plane shows that the wake is squeezed towards the model base more than that described for the longitudinal symmetry plane $\left(y^{*}=0.00\right)$. The momentum of the flow leaving the model underbody in this region is reduced due to its interaction with the flow passing along the sides of the model. As a consequence, the amount of upwash generated by the bottom slant is lower and the free stagnation point is shifted downwards. The streamlines leaving this point are now noticeably deflected towards the ground, forming an angle of almost $-19^{\circ}$ for $\phi_{b}=16^{\circ}$. The trailing vortices generated at the tips of the slant, however, squeeze the lower lobe towards the base and some of the streamlines leaving the bottom shear layer feed the top shear layer.

This last element is consistent with the presence of two separated horseshoe vortices, whose "tails" merge together in the upper part of the base. The first vortex runs parallel to the squared top trailing edge and near the vertical edges of the base its trailing legs align themselves with the direction of the onset flow. The second vortex, on the other hand, acts close to the bottom slant and the vertical sides of the base, creating the "U" shaped low pressure zone previously described before merging with the upper vortex. Similar structures in the wake topology and base pressure distribution can be found when a taper is applied to the top trailing edge leaving the opposite edge squared, as shown in Fig. $11 \mathrm{c}$ for the $\phi_{t}=16^{\circ}$. In this case, however, the downwash tends to prevail over the upwash; the streamlines close to the saddle point that are visible in the PIV plane at $z^{*}=0.67$ become concave, and the vortical structures previously described are turned upside down. 

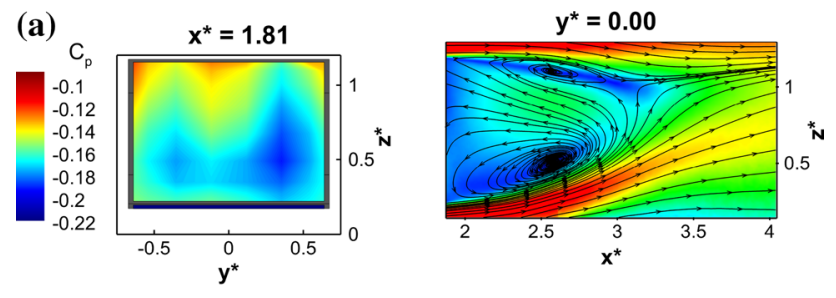

$z^{*}=0.67$
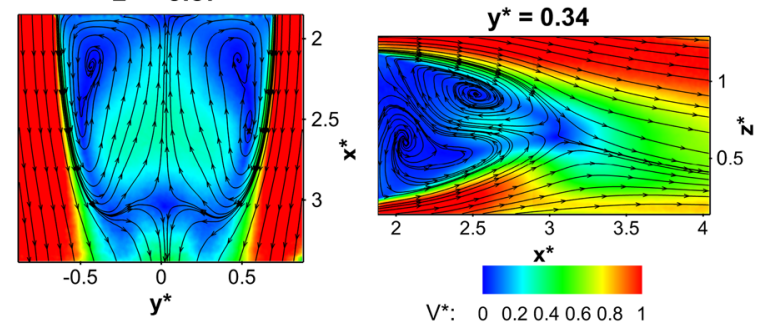

(b)
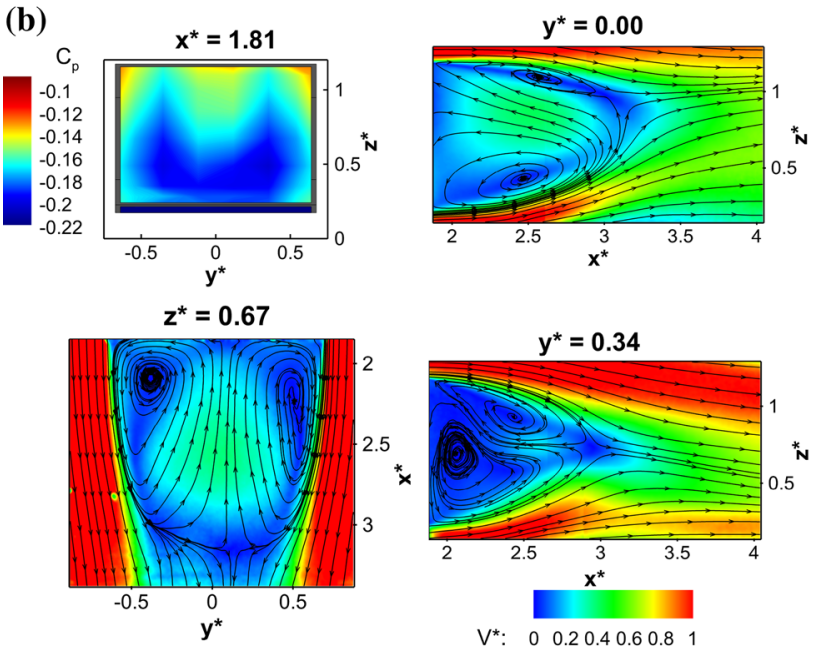

(c)
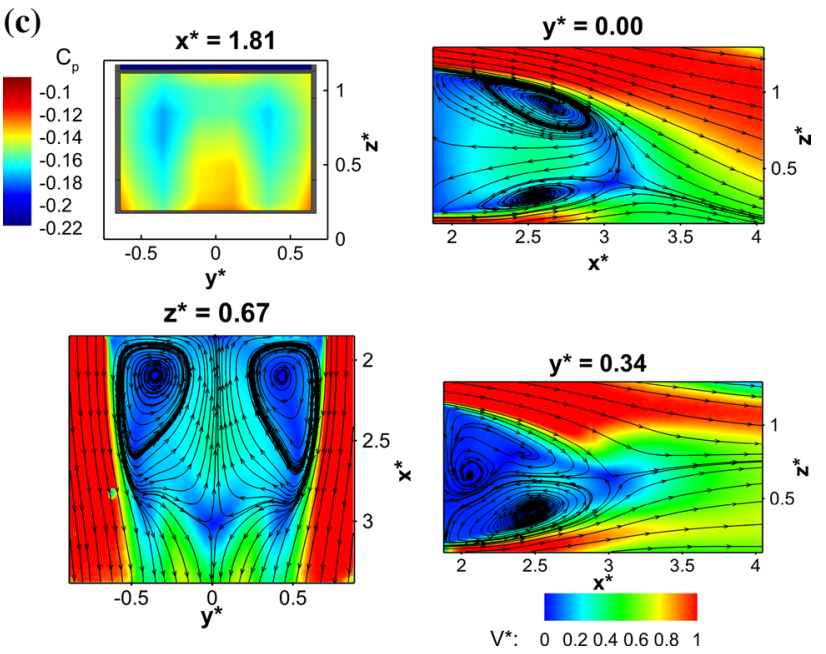

Fig. 11 Time-averaged fields for three different tapered configurations: a $\phi_{t}=0^{\circ}$ and $\phi_{t}=12^{\circ}, \mathbf{b} \phi_{t}=0^{\circ}$ and $\phi_{b}=20^{\circ}$, c $\phi_{t}=16^{\circ}$ and $\phi_{b}=0^{\circ}$. Clockwise from top left: $x^{*}=1.81$ base pressure distribution, $y^{*}=0.00$ PIV vertical mid-plane, $y^{*}=0.34$ PIV vertical offcentre plane, $z^{*}=0.67$ PIV horizontal mid-plane
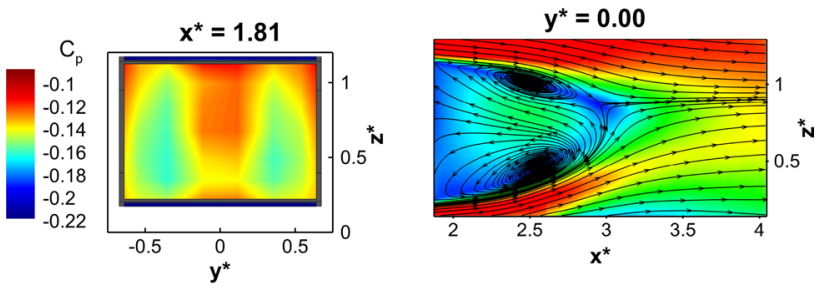

$z^{*}=0.67$
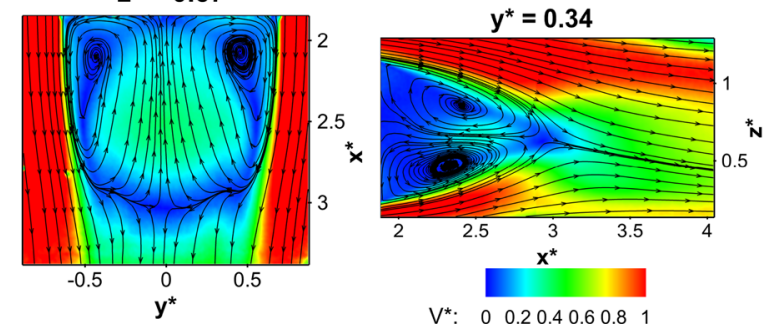

Fig. 12 Time-averaged fields for the low drag configuration $\left(\phi_{t}=12^{\circ}\right.$ and $\left.\phi_{b}=12^{\circ}\right)$. Clockwise from top left: $x^{*}=1.81$ base pressure distribution, $y^{*}=0.00$ PIV vertical mid-plane, $y^{*}=0.34$ PIV vertical off-centre plane, $z^{*}=0.67$ PIV horizontal mid-plane

The time-averaged base pressure and PIV results obtained for the low drag configuration are presented in Fig. 12. Looking at the vertical mid-plane $\left(y^{*}=0.00\right)$, the free stagnation point is located above mid-base height but the two shear layers have now a similar strength, so that the flow leaves the free stagnation point horizontally. This gives a near equal sizing to the vortex cores across all the PIV planes considered. The restored balance between upwash and downwash in the near-wake region leads to a better pressure recovery over the model base, as highlighted by the reduction of the size of the "U" shaped low pressure region.

\subsection{Sensitivity of bi-stability to taper angle}

The taper angles are having a clear effect on the overall topology of the wake and so an analysis of the unsteady characteristics of the wake was conducted to investigate the impact of these changes on bi-stability. The results related to the RMS of the fluctuation of the normalised pressure $C_{\mathrm{p}}$ are presented in Fig. 13. For the square-back case (Fig. 13a), considered here for reference, the switching of the rear stagnation point between two different positions is highlighted by the presence of the two distinct regions of highest fluctuation. The application of the tapers, besides yielding the changes in the time average wake topology and base pressure already discussed in Sect. 3.3, significantly affects the bi-stable nature of the wake itself (see Fig. 13b-d). The bi-stable region is modified in both extension and position by the upwash or downwash generated 
(a)
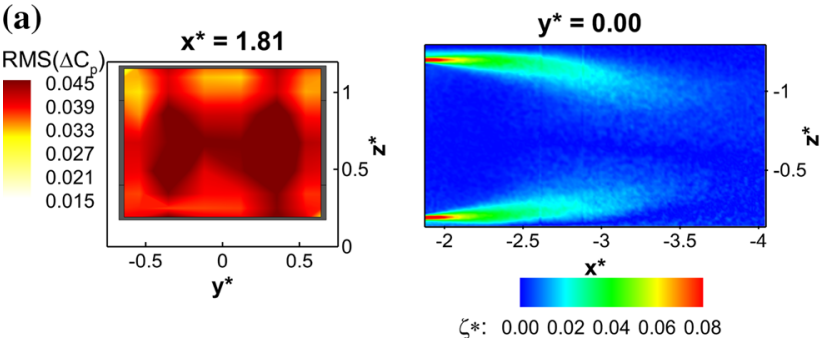

(c)
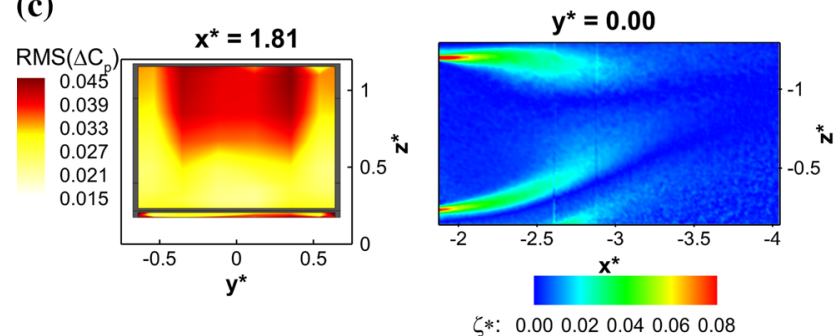

Fig. 13 Base pressure fluctuations and time-averaged vorticity magnitude (at $\left.y^{*}=0.00\right)$ for a square-back b low drag $\left(\phi_{t}=12^{\circ}\right.$ and $\left.\phi_{b}=12^{\circ}\right)$, c low lift $\left(\phi_{t}=0^{\circ}\right.$ and $\left.\phi_{b}=16^{\circ}\right)$ and $\mathbf{d}$ high lift

by the small slants, as shown by the deflection of the shear layers visible in the normalised vorticity fields $\zeta^{*}$ (where $\zeta^{*}=\left(H / V_{\infty}\right) \cdot \zeta$ ) included in Fig. 13. The region of highest RMS $\left(\Delta C_{\mathrm{p}}\right)$ is shifted upwards, compared to the square-back case, when a chamfer is applied to the bottom (Fig. 13c) and moves in the opposite direction when the same chamfer is applied to the top (Fig. 13d). At the same time, as the wake is deformed from its original torus and the distance between the top and bottom shear layers is shortened, the size of the high fluctuation region is reduced and a low fluctuation zone develops on the opposite side of the base. This is a direct consequence of the growth of the recirculating vortex acting on the same region. The role of the shear layers in determining the size and the position of the region with the highest values of $\operatorname{RMS}\left(\Delta C_{\mathrm{p}}\right)$ becomes even more clear when the lowest drag configuration is considered $\left(\phi_{t}=12^{\circ}, \phi_{b}=12^{\circ}\right.$, Fig. 13b). In this case, the area with the highest level of fluctuations is shifted back to the middle of the base as in the square-back, following the restoration of a symmetric condition between the two vertical recirculating structures. The reduced gap between the top and bottom shear layers, however, leads to a reduction of the extension of this region as well as a decrease in the magnitude of the fluctuations, which suggests that the wake is less bi-stable.

The tendency of the wake to switch between the two different states was then analysed by looking at the first POD mode extracted from the base pressure recordings as well as the two horizontal PIV planes. Results for selected configurations are illustrated in Fig. 14. For the square-back configuration (Fig. 14a), the first spatial mode extracted from the
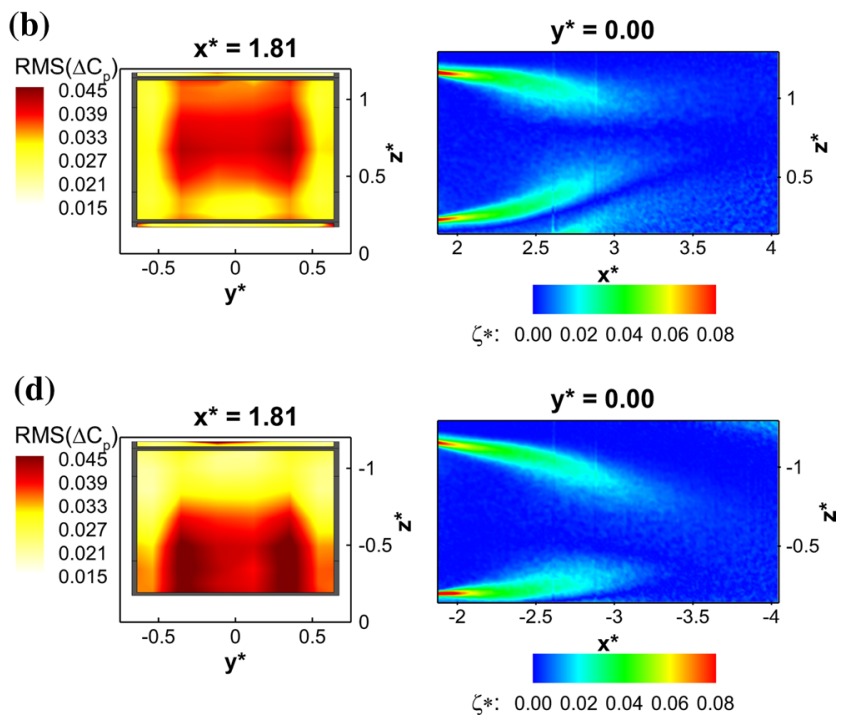

$\left(\phi_{t}=16^{\circ}\right.$ and $\left.\phi_{b}=0^{\circ}\right)$ configuration, a $\phi_{t}=0^{\circ}, \phi_{b}=0^{\circ}, \quad \mathbf{b}$ $\phi_{t}=12^{\circ}, \phi_{b}=12^{\circ}, \mathbf{c} \phi_{t}=0^{\circ}, \phi_{b}=16^{\circ}, \mathbf{d} \phi_{t}=16^{\circ}, \phi_{b}=0^{\circ}$

base pressure data shows the same left-to-right asymmetry demonstrated by Volpe et al. (2015) applying the same technique to the Ahmed body. Topologically, this mode is very similar to the RMS $\left(\Delta C_{\mathrm{p}}\right)$ distribution described in Fig. 13a for the same configuration. The same mode extracted from the horizontal PIV planes at the middle of the model base $\left(z^{*}=0.67\right)$ and close to the top trailing edge $\left(z^{*}=1.07\right)$ shows the existence of a well-defined vortical structure which extends for the entire width of the base and moves the core of the wake from one side to the other.

To further investigate the effects of taper angles on the wake bi-stability, a sweep of the bottom slant with a square top trailing edge was considered. The PDF distribution of the values of the first POD temporal coefficient extracted from the pressure data and the PIV horizontal mid-plane (at $z^{*}=0.67$ ) are presented in Fig. 15a, b. Using the pressure data, it is shown that as the bottom chamfer angle is increased, the bimodal PDF distributions gradually change with one of the two states more probable and a tendency to move towards a single near zero value. However, when the PDF distributions from the PIV data are considered a single peak can be seen for the configurations generating the highest amount of upwash $\left(\phi_{b}=12, \phi_{b}=16\right)$, although the general trend is still the same.

The tendency towards one single symmetric state yields a reduction of the fluctuating energy captured by the first POD mode. This can be seen by examining the plots presented in Fig. 15c for the pressure fields and in Fig. 15d for the PIV data on both horizontal planes. In all the cases, as the upwash increases and the bi-stability becomes less pronounced, the energy level of the first mode is reduced, until 

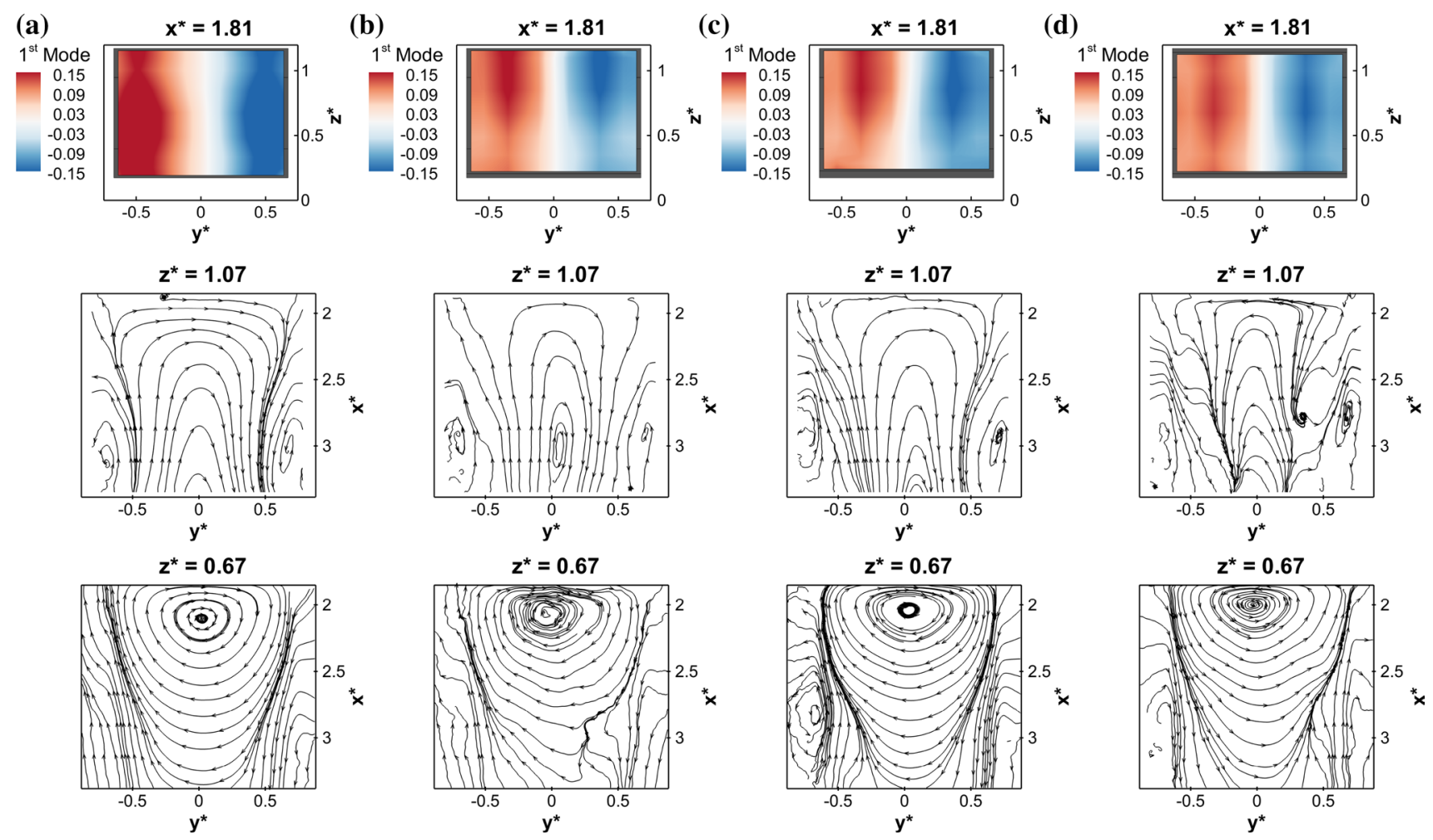

Fig. 14 Sensitivity of bi-stability to different bottom taper angles (1): first POD spatial mode extracted from base pressure distribution and two different PIV planes (at $z^{*}=0.67$ and $z^{*}=1.07$ ) for a square-

separation occurs on the bottom slant and the energy level increases again. However, as shown in Fig. 14b, c, the corresponding spatial mode maintains the left-to-right asymmetry seen for the square-back case.

An additional way to quantify the degree of bi-stability was found in the estimation of the average time between switches. This was done considering the first POD temporal coefficient extracted from the base pressure data, already used for separating the two states as explained in Sect. 3.1. The duration of each occurrence of a particular state was calculated and averaged over the number of occurrences. The results, reported in Fig. 15e, show for the square-back configuration a value of $t_{s}=9.94 \mathrm{~s}$, in good agreement with relationship $\left(t_{s} \approx 10^{3} \mathrm{H} / U_{\infty}\right)$ proposed by Grandemange et al. (2013b) and the results of Volpe et al. (2015), which reported $t_{s}=10.21 \mathrm{~s}$ for a square-back Ahmed body tested at the same Reynolds number $\operatorname{Re}_{H}=7.7 \times 10^{5}$. A reduction in the time between switches can be seen once a bottom taper is applied (weakening of the bi-stable behaviour). It reaches a minimum of $t_{s}=2.46 \mathrm{~s}$, before increasing again for the case with flow separation over the slant.

A further weakening of the bi-stable behaviour is noted for the lowest drag configuration (Fig. 14d), where the tendency towards the selection of one single symmetric state is shown in both the pressure and PIV data (Fig. 16a, b). back, b $\phi_{t}=0^{\circ}$ and $\phi_{b}=12^{\circ}$, c $\phi_{t}=0^{\circ}$ and $\phi_{b}=20^{\circ}$, d $\phi_{t}=12^{\circ}$ and $\phi_{b}=12^{\circ}$, a $\phi_{t}=0^{\circ}$ top, $\phi_{b}=0^{\circ}, \mathbf{b} \phi_{t}=0^{\circ}$ top, $\phi_{b}=12^{\circ}, \mathbf{c}$ $\phi_{t}=0^{\circ}$ top, $\phi_{b}=20^{\circ}, \mathbf{d} \phi_{t}=12^{\circ}$ top, $\phi_{b}=12^{\circ}$

The fluctuating energy captured by the first POD mode extracted from the pressure field is the lowest obtained across all the configurations considered in the present work (Fig. 16c). Furthermore, this is associated with the shortest time between switches, $t_{s}=1.77 \mathrm{~s}$. However, as for the bottom taper sweep, the left-to-right asymmetry can still be seen (Fig. 14d).

\subsection{Contribution of the base wall velocity to the drag}

The effects of the wake on the aerodynamic drag experienced by the entire model were studied by correlating the values of the time-averaged aerodynamic drag $C_{D}$, measured using the balance, with the values of the time-averaged rear drag $C_{D_{\text {Rear }}}$ calculated by integrating the base pressure. For the sake of simplicity, the rear drag is calculated including the contribution of the slants, according to Eq. 7. The results, presented in Fig. 17a, show the existence of a strong correlation between total and rear drag for all the configurations considered in this study, confirming that drag generated by the rearward facing surfaces is the dominant contribution (Littlewood and Passmore 2010) and that the rear drag drives the variations of $C_{d}$ recorded for different combinations of the horizontal slants (Perry et al. 2015). 
(a)

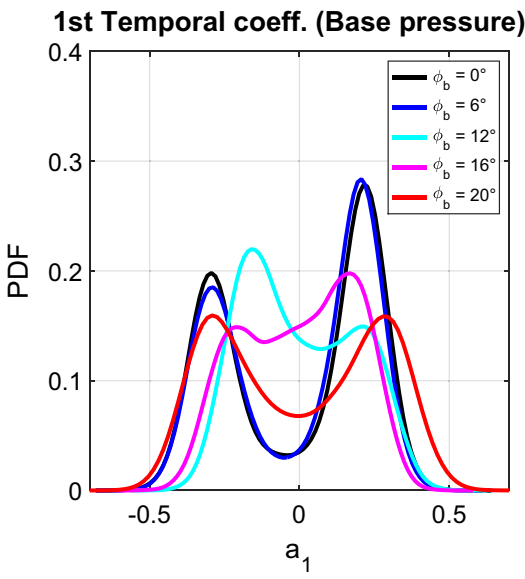

(c)

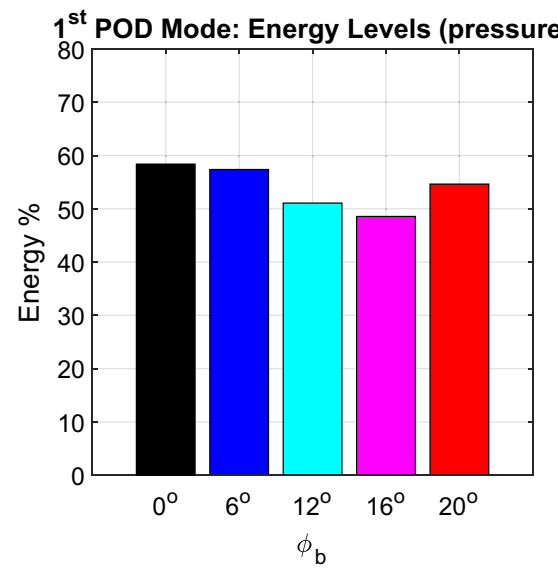

(b)

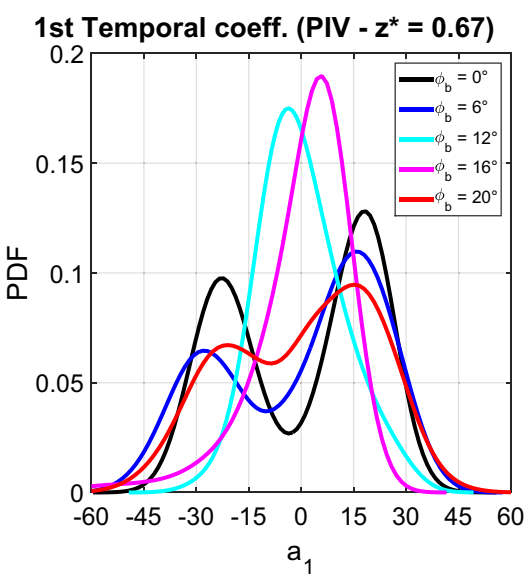

(e) (d)

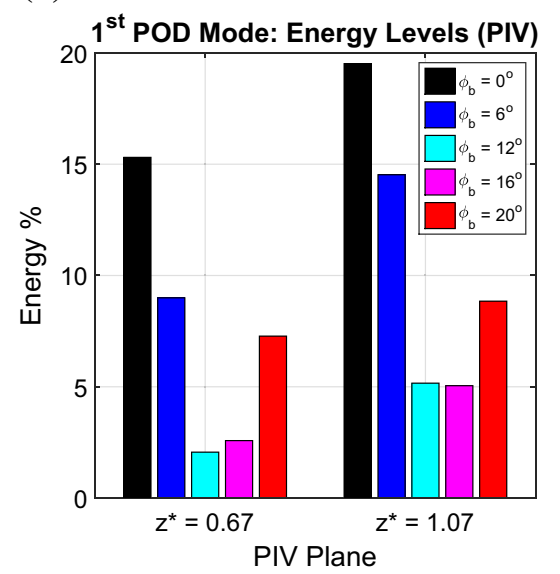

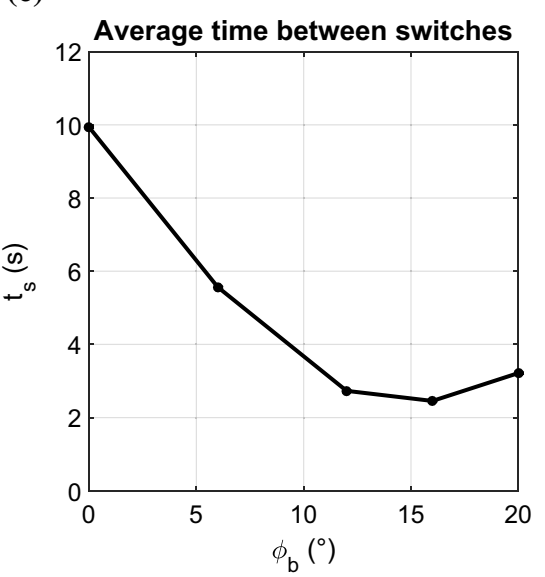

Fig. 15 Sensitivity of bi-stability to different bottom taper angles (2): PDF distributions for the first POD temporal mode (a base pressure data, b PIV horizontal mid-plane); c fluctuating energy captured by the first POD mode extracted from the pressure data; d fluctuating energy captured by the first POD mode along two different horizontal PIV planes (at $z^{*}=0.67$ and $z^{*}=1.07$ ); e averaged time between switches (a)

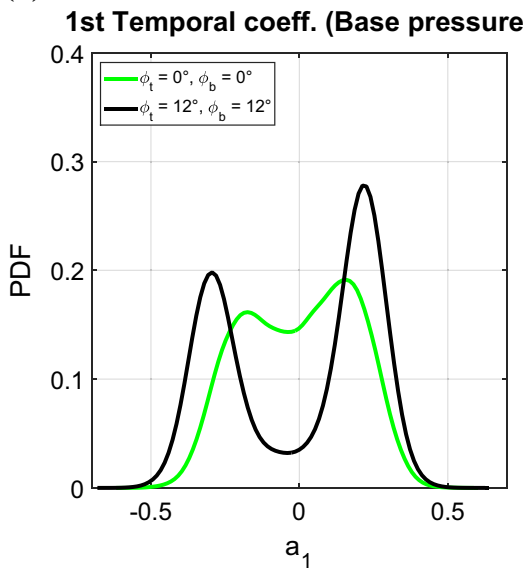

(b)

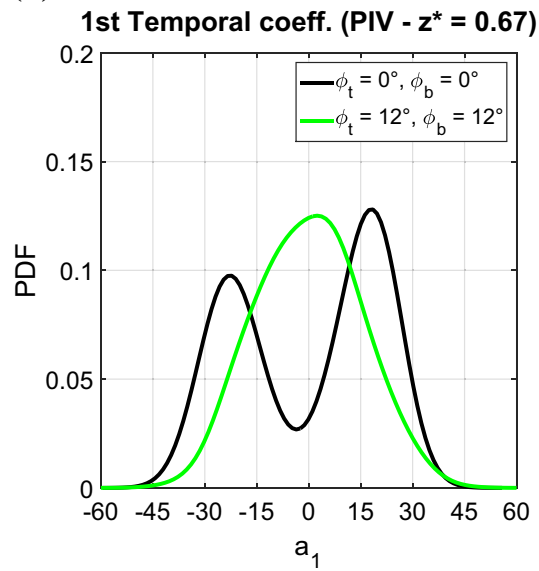

(c)

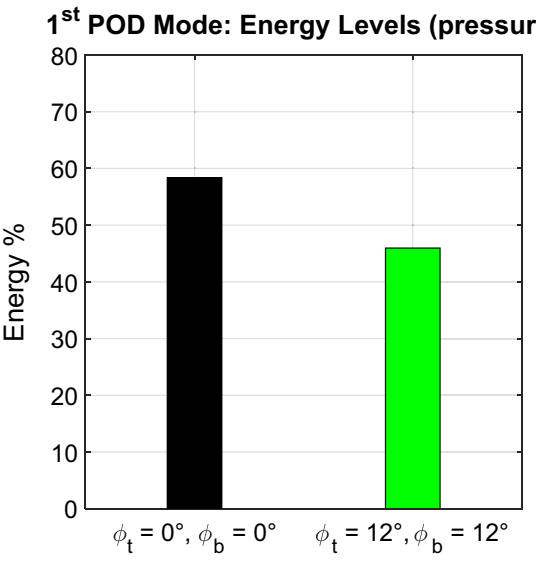

Fig. 16 PDF distributions of the values of the first POD temporal mode for the square-back and the low drag configuration extracted from a the base pressure data, $\mathbf{b}$ the PIV horizontal mid-plane. c turbulent energy captured by the first POD mode extracted from the pressure data in the two cases 
(a)

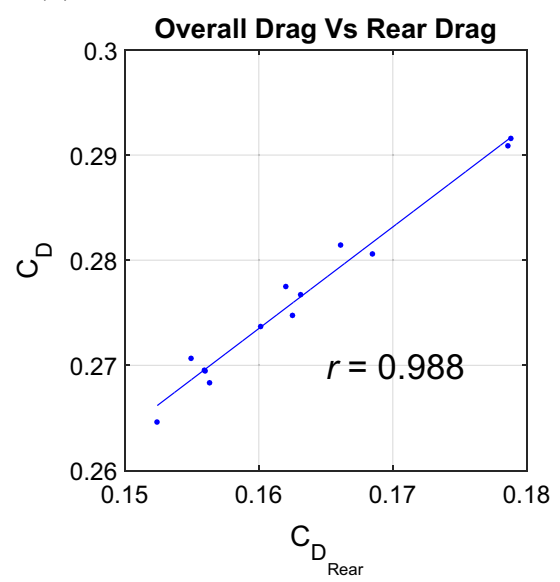

(b)

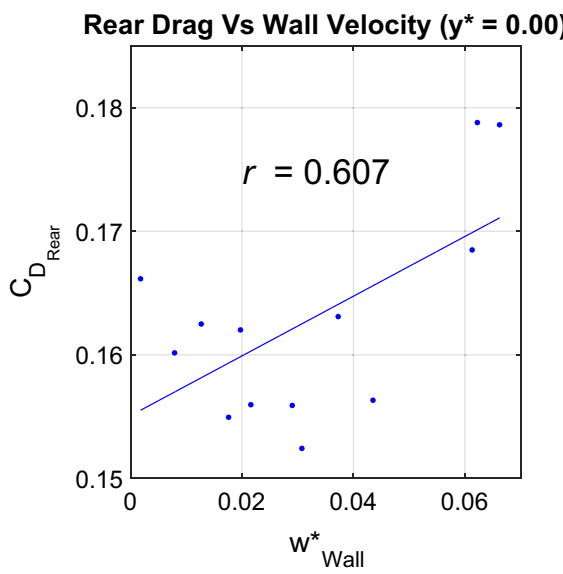

(c)

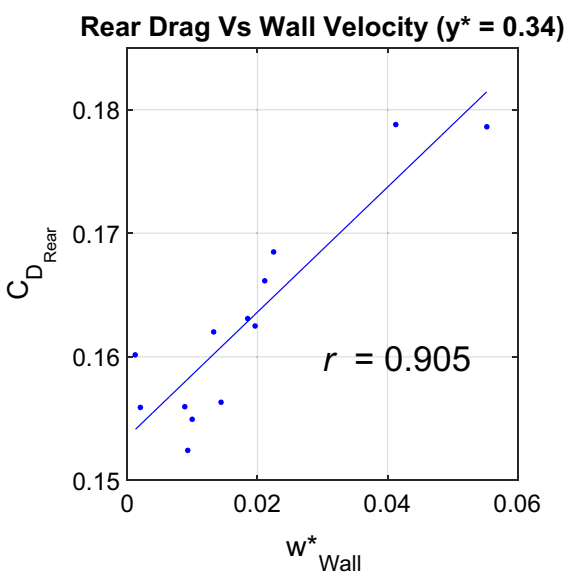

Fig. 17 a Correlation between aerodynamic drag and pressure drag; correlation between pressure drag and tangential wall velocity for b $y^{*}=0.00$ and $\mathbf{c} y^{*}=0.34$

The link between the changes in the wake topology and the different base pressure distributions qualitatively described in Sect. 3.3 was then characterised by considering the normalised value of the magnitude of the tangential wall velocity $w_{\text {Wall }}^{*}$, defined as the spatial average of the time-averaged value of the vertical component of the velocity $V_{z}$ estimated using the vertical PIV planes taken at $y^{*}=0.0$ and $y^{*}=0.34$, considering a domain $S_{\text {wake }}$ defined as the region delimited by the top and the bottom shear layers and extending in the streamwise direction for $\delta x^{*}=0.1 \mathrm{~h}$ from the model base, according to Eq. 10:

$w_{\text {Wall }}^{*}=\frac{1}{V_{\infty}}\left|\frac{1}{S_{\text {wake }}} \iint_{S_{\text {wake }}} V_{z} \cdot \mathrm{d} S\right|$.

Since the flow field captured by the vertical off-centre plane has proven to be affected by the presence of bi-stability, the state average fields were used for this plane rather than the canonical averaged ones. This eliminated any distortion in the results, as the state average contains equal weighting for the two states. The results for the vertical mid-plane $\left(y^{*}=0.00\right.$, Fig. 17b) show the existence of a weak correlation between $w_{\text {Wall }}^{*}$ and $C_{d_{\text {Rear }}}(r=0.607)$, suggesting that a single centreline 2D PIV plane is not sufficient to fully characterise the topology of the highly three-dimensional wake generated by such a bluff body (Grandemange et al. 2013c) and (Perry et al. 2016). However, when a plane located closer to the low pressure region described in Sect. 3.3 is considered (as for $y^{*}=0.34$ ), a strong correlation between $w_{\text {Wall }}^{*}$ and $C_{d_{\text {Rear }}}$ is found $(r=0.905$, Fig. $17 \mathrm{c})$. This element suggests the existence of a direct relation between the low pressure acting over the model base and the vortex structures shed in the near-wake region. As a consequence of the predominance of either the upwash or the downwash, the vortical structure close to the lower or the upper portion of the model base, respectively, expands at the expense of the recirculation acting on the opposite side. This yields an increase of the magnitude of the velocity tangent to the base, which results in a reduction of the static pressure acting in the same region. When the vortical structures are squeezed towards the base, as in the case of the plane located at $y^{*}=0.34$, this relation becomes even stronger, in agreement with the results of the numerical simulations performed by Bruneau et al. (2010).

This mechanism would explain the drag reduction obtained in Littlewood et al. (2011), where applying small horizontal slats to the lower half of the base of the same model considered here produced an increase of $C_{\mathrm{p}}$. The high number of points around the lowest values of $w_{\mathrm{Wall}}^{*}$ in the plot presented in Fig. 17c also provides some insight into the contribution to the rear drag of other wake features, such as the length of the wake and its tendency to develop a bi-stable behaviour. In fact, both the square-back and the low drag configurations are in this region of the plot, since they both show a value of $w_{\text {Wall }}^{*}$ close to zero. However, in the latter configuration, the wake appears to be shorter as well as less bi-stable than in the squareback case and in fact there is a clear connection between these two elements, i.e. a longer wake is more affected by bi-stability (Sect. 3.4), which seems to suggests that limiting the bi-stable behaviour might have a positive effect on the rear drag, in analogy with the results of Grandemange et al. (2015) studying the effects of similar tapers applied to a passenger car scale Ahmed body with rotating wheels and Evrard et al. (2016) looking at the effects produced by base cavities. 


\section{Summary and conclusions}

An experimental programme, consisting of balance measurements, base pressures and 2D PIV has been conducted to study the aerodynamic effects produced by short tapered sections applied to the trailing edge of the roof and underside of a simplified passenger car model.

The existence of wake bi-stability, already studied by Grandemange et al. (2013c), Volpe et al. (2015) and Evrard et al. (2016), has been proved for a model with a more representative front-end geometry than the Ahmed body, and the structure of the two symmetry-breaking states has been characterised. The PIV and base pressure fields, conditionally averaged using POD, suggest the existence of two vortical structures: a "slow" horseshoe vortex, extending in the vertical direction and causing a low pressure region that extends for almost half of the model base, and a "faster" "C" shaped horseshoe vortex acting on the opposite side of the base, connecting the top and the bottom shear layers. The toroidal structure, reported by Krajnovic and Davidson (2003) and Rouméas et al. (2009a) for similar geometries, is obtained only when the average of the two states is considered.

The effects of bi-stability on the lateral force and the related moments have been analysed by performing a yaw sweep for $-2^{\circ} \leq \psi \leq+2^{\circ}$ in the square-back configuration. The plots show a nonlinear region for the coefficients of Side force, Roll and Yaw moment that is associated with the presence of bi-stability, confirming the high sensitivity of bi-stability to small variations of the yaw angle (Volpe et al. 2015; Pavia et al. 2016), its effect on the side force (Evrard et al. 2016) and also on the rolling and yawing moments.

The application of high aspect ratio tapers to the top and bottom edges of the model base has proved to be effective in producing measurable changes in the time-averaged wake topology and base pressure distribution, which ultimately result in variations of the aerodynamic drag experienced by the entire model. In particular, an increase in drag occurs when the application of the taper leads to the creation of a strong upwash or downwash that breaks down the horizontal symmetry between the vortical structures seen for the square-back configuration. In these conditions, the flow field is dominated by the presence of a " $U$ " shaped horseshoe vortex, acting close to the taper plus a second horseshoe vortex shed from opposite horizontal trailing edge. As the " $U$ " shaped vortex interacts with the surface of the base, a low pressure region, responsible for an increase in drag, is formed as the vertical legs of the vortex are squeezed towards the base by the local upwash/downwash generated by a trailing vortex pair originating from the tips of the slants.
As the gap between the top and bottom shear layers decreases, the tendency of the wake to develop a bi-stable behaviour is reduced, until almost disappearing for the configuration featuring a $12^{\circ}$ slant on both of the trailing edges. The weakening of the wake bi-stability, together with the shortening of length of the wake, is found to be the reason for the drag decrease compared to the square-back case.

Acknowledgments The authors would like to thank Jaguar Land Rover and the EPSRC for their financial support. Thanks are also due to Mr. Rob Hunter, Mr. David Cooper and Mr. Nigel Lines for their excellent work in manufacturing the models and keeping the test facility always in optimal conditions.

Open Access This article is distributed under the terms of the Creative Commons Attribution 4.0 International License (http://creativecommons.org/licenses/by/4.0/), which permits unrestricted use, distribution, and reproduction in any medium, provided you give appropriate credit to the original author(s) and the source, provide a link to the Creative Commons license, and indicate if changes were made.

\section{References}

Aider J-L, Beaudoin J-F, Wesfreid JE (2010) Drag and lift reduction of a 3D bluff-body using active vortex generators. Exp Fluids 48(5):771-789

Brown YI, Windsor S, Gaylard A (2010) The effect of base bleed and rear cavities on the drag of an SUV. Technical report, SAE technical paper

Bruneau C-H, Creusé E, Depeyras D, Gilliéron P, Mortazavi I (2010) Coupling active and passive techniques to control the flow past the square back Ahmed body. Comput Fluids 39(10):1875-1892

Duell EG, George A (1993) Measurements in the unsteady near wakes of ground vehicle bodies. Technical report, SAE technical paper

Duell EG, George A (1999) Experimental study of a ground vehicle body unsteady near wake. Technical report, SAE technical paper

Evrard A, Cadot O, Herbert V, Ricot D, Vigneron R, Délery J (2016) Fluid force and symmetry breaking modes of a 3D bluff body with a base cavity. J Fluids Struct 61:99-114

Grandemange M, Gohlke M, Cadot O (2013a) Bi-stability in the turbulent wake past parallelepiped bodies with various aspect ratios and wall effects. Phys Fluids (1994 Present) 25(9):95-103

Grandemange M, Gohlke M, Cadot O (2013b) Turbulent wake past a three-dimensional blunt body. Part 1 . Global modes and bi-stability. J Fluid Mech 722:51-84

Grandemange M, Mary A, Gohlke M, Cadot O (2013c) Effect on drag of the flow orientation at the base separation of a simplified blunt road vehicle. Exp Fluids 54(5):1-10

Grandemange M, Gohlke M, Cadot O (2014) Turbulent wake past a three-dimensional blunt body. Part 2. Experimental sensitivity analysis. J Fluid Mech 752:439-461

Grandemange M, Cadot O, Courbois A, Herbert V, Ricot D, Ruiz T, Vigneron R (2015) A study of wake effects on the drag of Ahmeds squareback model at the industrial scale. J Wind Eng Ind Aerodyn 145:282-291

Johl G (2010) The design and performance of a $1.9 \mathrm{mx} 1.3 \mathrm{~m}$ indraft wind tunnel. $\mathrm{PhD}$ thesis, Guru Johl

Kim J, Hahn S, Kim J, Lee D-K, Choi J, Jeon W-P, Choi H (2004) Active control of turbulent flow over a model vehicle for drag reduction. J Turbul 5(019):1-12 
Krajnovic S, Davidson L (2003) Numerical study of the flow around a bus-shaped body. J Fluids Eng 125(3):500-509

Littlewood R, Passmore M (2010) The optimization of roof trailing edge geometry of a simple square-back. Technical report, SAE technical paper

Littlewood R, Passmore M (2012) Aerodynamic drag reduction of a simplified squareback vehicle using steady blowing. Exp Fluids 53(2):519-529

Littlewood R, Passmore M, Wood D (2011) An investigation into the wake structure of square back vehicles and the effect of structure modification on resultant vehicle forces. Technical report, SAE technical paper

Lumley JL (1967) The structure of inhomogeneous turbulent flows. In: Atmospheric turbulence and radio wave propagation, pp 166-178

Passmore MA, Spencer A, Wood D, Jowsey L, Newnham P (2010) The application of particle image velocimetry in automotive aerodynamics. Technical report, SAE technical paper

Pavia G, Passmore M, Gaylard A (2016) Influence of short rear end tapers on the unsteady base pressure of a simplified ground vehicle. Technical report, SAE technical paper

Perry A-K, Passmore M (2013) The impact of underbody roughness on rear wake structure of a squareback vehicle. Technical report, SAE technical paper

Perry A-K, Passmore M, Finney A (2015) Influence of short rear end tapers on the base pressure of a simplified vehicle. SAE Int $\mathbf{J}$ Passeng Cars Mech Syst 8(2015-01-1560):317-327
Perry A-K, Almond M, Passmore M, Littlewood R (2016) The study of a bi-stable wake region of a generic squareback vehicle using tomographic PIV. SAE Int J Passeng Cars Mech Syst 9(2016-01-1610):743-753

Rouméas M, Gilliéron P, Kourta A (2009a) Analysis and control of the near-wake flow over a square-back geometry. Comput Fluids 38(1):60-70

Rouméas M, Gilliéron P, Kourta A (2009b) Drag reduction by flow separation control on a car after body. Int J Numer Methods Fluids 60(11): 1222-1240

SAE (2010) Surface vehicle recommended practice. Technical report J1594, SAE international

Sirovich L (1987) Turbulence and the dynamics of coherent structures. Part I: coherent structures. Q Appl Math 45(3):561-571

Thacker A, Aubrun S, Leroy A, Devinant P (2010) Unsteady analyses of the flow separation on the rear window of a simplified ground vehicle model. AIAA paper, 4569

Van Raemdonck G, Van Tooren M (2008) Time averaged phenomenological investigation of a wake behind a bluff body. In: Bluff body aerodynamics and application VI conference, Milan

Volpe R, Devinant P, Kourta A (2015) Experimental characterization of the unsteady natural wake of the full-scale square back Ahmed body: flow bi-stability and spectral analysis. Exp Fluids 56(5):1-22 